\title{
A Survey on Inverse Problems for Applied Sciences
}

\author{
Fatih Yaman, ${ }^{1}$ Valery G. Yakhno, ${ }^{2}$ and Roland Potthast ${ }^{3,4}$ \\ ${ }^{1}$ Institut für Theorie Elektromagnetischer Felder, Technische Universität Darmstadt, 64289 Darmstadt, Germany \\ ${ }^{2}$ Department of Electrical and Electronics Engineering, Dokuz Eylul University, 35160 İzmir, Turkey \\ ${ }^{3}$ Department of Mathematics, University of Reading, Whiteknights P.O. Box 220, Reading RG6 6AX, UK \\ ${ }^{4}$ German Meteorological Service, Deutscher Wetterdienst Research and Development, \\ Head Division FE 12 (Data Assimilation) Frankfurter Straße 135, 63067 Offenbach, Germany \\ Correspondence should be addressed to Fatih Yaman; yaman@temf.tu-darmstadt.de
}

Received 19 March 2013; Accepted 16 June 2013

Academic Editor: Baocang Ding

Copyright (C) 2013 Fatih Yaman et al. This is an open access article distributed under the Creative Commons Attribution License, which permits unrestricted use, distribution, and reproduction in any medium, provided the original work is properly cited.

\begin{abstract}
The aim of this paper is to introduce inversion-based engineering applications and to investigate some of the important ones from mathematical point of view. To do this we employ acoustic, electromagnetic, and elastic waves for presenting different types of inverse problems. More specifically, we first study location, shape, and boundary parameter reconstruction algorithms for the inaccessible targets in acoustics. The inverse problems for the time-dependent differential equations of isotropic and anisotropic elasticity are reviewed in the following section of the paper. These problems were the objects of the study by many authors in the last several decades. The physical interpretations for almost all of these problems are given, and the geophysical applications for some of them are described. In our last section, an introduction with many links into the literature is given for modern algorithms which combine techniques from classical inverse problems with stochastic tools into ensemble methods both for data assimilation as well as for forecasting.
\end{abstract}

\section{Introduction}

Finding causes from the knowledge of their effects, which actually constitutes the idea of solving inverse problems, is necessary for the identifications of practical applications in different critical areas such as mine detection, medical imaging, remote sensing, nondestructive testing, and geophysical explorations. It is also a crucial ingredient of forecasting in basic areas such as weather prediction or projections for climate change.

However, small changes in the effects might result in large differences in the causes, or the same effect might be obtained from more than one cause. Therefore, it is difficult or sometimes impossible to find actual reason uniquely by observing only effects. This ill-posed fundamental characteristic of inverse problems influences the complexity and features of their solutions dramatically [1-6].

It is a generally accepted view that the first mathematical investigation of inverse problems is the study of Abel's on a mechanical problem for finding the curve of an unknown path in 1826. On the other hand, the invention of radar and sonar during the Second World War inspired researchers to focus especially on inverse scattering problems whose aims are not only to determine locations of the targets from the transmitter/receiver antennas but also to construct their detailed images. This motivation induced the progress in developing new reconstruction methods and their extensions to other research branches such as nondestructive testing, biomedical imaging, seismology, and atmospheric profile inversions. This agenda has led to a large number of methods and tools since 1980 , with a continuing flow of new ideas into the field.

Several inversion methods in scattering and tomography have been suggested in the 1980s and 1990s, with qualitative methods arising since around 1996. Since 2000, an increased interest in the treatment of sparsity has led to new regularization tools and new approaches. Also, the need to carry out inversion as a part of dynamical systems and forecasting, also called data assimilation, is leading to an increasing synergy of filtering methods from stochastics and inversion tools from 
regularization theory. Since around 2000, multiphysics-based methods have become very popular, leading to increased insight for example into the human body or the atmosphere. Nowadays the advent of powerful computers and high technologies made it possible to evaluate and process large volume of data for finding sufficiently accurate solutions of practical inverse problems. For a more detailed information into the different areas of inverse problems we refer to [7-20].

The plan of the paper is as follows. A short summary to the inverse problems in the historical perspective has just been reported in the introduction section. The following two sections are devoted for the presentation of the mathematical basics used in the solution of well-known inverse problems for acoustic, electromagnetic, and elastic waves. Clearly, we cannot aim to cover their entire literature with this survey but describe some interesting and important lines of development. Afterwards, some of important inversionbased practical applications are introduced, and as a selected topic, inverse problems for the neural field equation is described in details. In the final section, conclusions and concluding remarks are given.

\section{Inverse Problems for Time-Harmonic Acoustic and Electromagnetic Waves}

In this section, we focus on the mathematical investigation of inverse problems whose aims are to reconstruct geometrical and/or physical properties of penetrable/impenetrable objects from the knowledge of scattered acoustic or electromagnetic waves at certain measurement points. Physically speaking, identifications of the unknown and inaccessible objects with certain waves are the main problems of the nondestructive testing, radar/sonar applications, tumor detection, and so forth.

Let us assume a time-harmonic plane wave $u^{i}=e^{i(k x \cdot d-\omega t)}$, which was insonificated by a sufficiently far acoustic source operating at a constant frequency $\omega$. The acoustic wave propagates in the direction $d$, with a speed of sound $c$, in a homogeneous medium having a wave number $k=$ $\omega / c$. In this background medium, we consider a bounded penetrable/impenetrable scatterer with support given by a domain $D \in \mathbb{R}^{m}(m=2,3)$ and define $v$ as the unit outward normal to the boundary $\partial D$.

The scattered field $u^{s}$, which appears from the interaction of the incident wave with the obstacle, satisfies the Sommerfeld radiation condition at infinity

$$
\lim _{r \rightarrow \infty} r^{(m-1) / 2}\left(\frac{\partial u^{s}}{\partial r}-i k u^{s}\right)=0, \quad r=|x|,
$$

and ensures that the scattered wave has the form of an outgoing wave. Furthermore, the so-called far-field pattern $u_{\infty}$ can be obtained from the asymptotic behavior of the scattered wave such that

$$
\begin{array}{r}
u^{s}(x)=\frac{e^{i k r}}{r^{(m-1) / 2}}\left\{u_{\infty}(\widehat{x})+O\left(\frac{1}{r}\right)\right\}, \\
\widehat{x}=\frac{x}{|x|}, \quad r=|x| \rightarrow \infty .
\end{array}
$$

The total field $u$ is simply the superposition of incident and scattered fields $u=u^{i}+u^{s}$, which satisfies the homogeneous Helmholtz equation in the exterior domain of the obstacle

$$
\Delta u+k^{2} u=0 \quad \text { in } \mathbb{R}^{m} \backslash \bar{D} \quad \text { for } \quad(m=2,3)
$$

and the boundary condition depending on scatterer type. Commonly used boundary conditions in the literature are given in the following.

The Dirichlet boundary condition:

$$
u=0 \quad \text { on } \partial D
$$

the Neumann boundary condition:

$$
\frac{\partial u}{\partial \nu}=0 \quad \text { on } \partial D
$$

the impedance boundary condition:

$$
u+\frac{\eta}{i k} \frac{\partial u}{\partial v}=0 \quad \text { on } \partial D
$$

the conductive boundary condition:

$$
u_{0}=u, \quad \frac{\partial u}{\partial v}-\frac{\partial u_{0}}{\partial v}=\lambda u \text { on } \partial D .
$$

Parameters $\eta$ and $\lambda$ are the impedance and conductivity functions, respectively. In latest equation, $u_{0}$ is the total field inside of the scatterer, and this boundary condition can be considered as a more general form of the transmission conditions.

On the other hand, in order to model acoustic scattering by an inhomogeneous medium, it is sufficient to replace (3)(7) with

$$
\Delta u+k^{2} n(x) u=0 \quad \text { in } \mathbb{R}^{m} \text { for }(m=2,3),
$$

where $n(x)=c^{2} / c_{i}^{2}(x)+i \sigma_{i}(x)$ is the refractive index, $c$ is the sound speed in the homogeneous medium, $c_{i}(x)$ is the speed of sound in the inhomogeneous medium, and $\sigma_{i}(x) \geq 0$ models the absorption.

Analogically one can also consider a scattering problem for electromagnetic waves assuming that the electric and magnetic field components of the incident plane wave are given by

$$
\begin{gathered}
E^{i}(x, t)=i k(d \times p) \times d e^{i(k x \cdot d-\omega t)}, \\
H^{i}(x, t)=i k d \times p e^{i(k x \cdot d-\omega t)},
\end{gathered}
$$

where $k=\omega \sqrt{\epsilon \mu}$ is the wave number and $\epsilon$ and $\mu$ are the electric permittivity and magnetic permeability of the host medium, respectively. Here, $\omega$ represents the frequency of the wave source, $d$ is the direction, and $p$ is the polarization of the electromagnetic wave. Then, we consider that the wave interacts with a $3 \mathrm{D}$ obstacle $D \in \mathbb{R}^{3}$ in the homogeneous medium and that scattered electromagnetic fields $\left(E^{s}, H^{s}\right)$ occur similar to the previous case. However, in electromagnetics, the scattered field has to satisfy the Silver-Müller radiation condition

$$
\lim _{r \rightarrow \infty}\left(H^{s} \times x-r E^{s}\right)=0 .
$$


The far field pattern of the corresponding scattered field can be obtained by

$$
\begin{aligned}
E^{\mathcal{S}}(x) & =\frac{e^{i k r}}{r}\left\{E_{\infty}(\widehat{x})+O\left(\frac{1}{r}\right)\right\}, \\
\widehat{x} & =\frac{x}{|x|}, \quad r=|x| \longrightarrow \infty .
\end{aligned}
$$

Furthermore, the total electric field $E=E^{i}+E^{s}$ and the total magnetic field $H=H^{i}+H^{s}$ in the medium satisfy the Maxwell equations

$$
\operatorname{curl} E-i k H=0, \quad \operatorname{curl} H+i k E=0 \quad \text { in } \mathbb{R}^{3} \backslash \bar{D}
$$

and the chosen boundary conditions on the surface of the scatterer. The boundary conditions which were presented for acoustic waves are now given for electromagnetic waves in (13)-(16).

The Dirichlet-type perfect conductor boundary condition:

$$
v \times E=0 \quad \text { on } \partial D,
$$

the Neumann-type boundary condition:

$$
v \times H=0 \quad \text { on } \partial D,
$$

the impedance boundary condition:

$$
\nu \times E+\frac{\eta}{i k}(\nu \times(\nu \times H))=0 \quad \text { on } \partial D,
$$

the conductive boundary condition:

$$
\begin{gathered}
\nu \times E_{0}=\nu \times E, \\
\nu \times(\nu \times H)-\xi \nu \times\left(\nu \times H_{0}\right)=\lambda(\nu \times E)
\end{gathered}
$$

on $\partial D$.

The parameter $\xi$ in the last equation is the ratio of the wave numbers $\xi=k_{0} / k$, where $k_{0}$ is the wave number of the object's interior domain.

For the case of modeling scattering of electromagnetic waves by an inhomogeneous medium, (12)-(16) are replaced by

$$
\operatorname{curl} E-i k H=0, \quad \operatorname{curl} H+i k n(x) E=0 \quad \text { in } \mathbb{R}^{3},
$$

where $n(x)=1 / \epsilon\left(\epsilon_{i}+i \sigma_{i}(x) / \omega\right)$ is the refractive index, $\epsilon$ is the permittivity of the homogeneous medium, $\epsilon_{i}$ is the permittivity of the inhomogeneous medium, $\sigma_{i}(x)$ is the conductivity, and $\omega$ is the frequency of the wave.

We recommend to the readers the books $[9,21-23]$ for the detailed investigations of the electromagnetic and acoustic waves with the same notation.

2.1. Boundary Reconstruction Problems of Acoustic Waves. In this section, our main concern is to present mathematical treatments of some available methods for reconstructing shapes of obstacles from the knowledge of scattered field.
Solution of this type of problems with acoustic waves are important from practical point of view since the real life experiments with acoustic waves are easier, safer, and lower in cost.

To provide a simpler mathematical presentation, we consider a two dimensional simply connected object $D \in \mathbb{R}^{2}$ having the Dirichlet condition on its boundary $\Gamma$. It is further assumed that the incident acoustic wave $u^{i}$, which interacts with the object, and scattered near/far fields are given. This inverse problem is nonlinear due to the mathematical relation between the scattered wave and the shape of the cylinder, and it is ill-posed since the determination of $\Gamma$ does not depend continuously on the scattered field [18]. For the solution of this type of inverse problems, there are a variety of methods of which we present a short list in the following without claiming to cover the entire literature.

\section{Iterative Methods}

(i) Landweber iterations [24, 25].

(ii) Regularized Newton [22, 26-50].

(iii) Newton-Kantorovich [51, 52].

\section{Decomposition Methods}

(i) Colton-Monk [53-55].

(ii) Kirsch-Kress [56-58].

(iii) Angell-Kleinmann-Roach [59-61].

(iv) Hybrid method [62-67].

(v) Potthast's point source method [23, 68, 69].

Probe and Sampling Methods

(i) Linear sampling [70-74].

(ii) Factorization $[75,76]$.

(iii) Singular sources $[19,23,77]$.

(iv) Probe method [78, 79].

(v) Enclosure method [80, 81].

(vi) No-response test [82-84].

In the first group of methods $[24,52]$, the inverse obstacle problem is considered as an ill-posed nonlinear operator equation,

$$
A\left(\Gamma_{0}\right)=u_{\infty},
$$

for an initial boundary $\Gamma_{0}$. Often, it is assumed to be a star-like parametrization $\Gamma_{0}=\{z(t):=r(t)(\cos t, \sin t): t \in[0,2 \pi)\}$. Here, the operator $A: \Gamma \rightarrow u_{\infty}$ is actually defined to map the boundary $\Gamma$ of the scatterer onto the far field $u_{\infty}$. Newton's method to solve a nonlinear equation employs successive linearization; that is, we replace (18) by an equation for the update $h_{n}$

$$
A\left(z_{n}\right)+A^{\prime}\left(z_{n}\right) h_{n}=u_{\infty}
$$

where

$$
z_{n+1}=z_{n}+h_{n}, \quad n=0,1,2, \ldots
$$


leading to a sequence of linear equations (19) and updates (20) with starting value $z_{0}:=\Gamma_{0}$. Equation (19) is ill-posed and needs to be regularized. As a simple option, Tikhonov's regularization with regularization parameter $\alpha>0$ can be applied, leading to

$$
\alpha h_{n}+\left[A\left(z_{n}\right)^{\prime}\right]^{*} A^{\prime}\left(z_{n}\right) h_{n}=\left[A\left(z_{n}\right)^{\prime}\right]^{*}\left[u_{\infty}-A\left(z_{n}\right)\right]
$$

in each iteration step $n=0,1,2, \ldots$. Iterative methods for illposed equations need to be stopped after a finite number of iterations to keep the ill-posedness under control; compare, for example, $[23,85]$.

The main idea of the decomposition methods is to split full nonlinear shape reconstruction problem given by (18) into a linear ill-posed equation which is solved first and a nonlinear well-posed equation to be solved in a second step.

In order to discuss the Kirsch-Kress method [56-58] via potential approach let us employ an initial boundary $\Gamma_{0}$ as an approximation of the actual boundary $\Gamma$. We assume that $\Gamma_{0}$ is in the interior of the true scatterer.

In this case, the approximate total field $\tilde{u}(x)$ can be approximated by the sum of the incident wave $u^{i}$ and a singlelayer potential,

$$
\widetilde{u}(x)=u^{i}(x)+\int_{\Gamma_{0}} \Phi(x, y) \varphi(y) d s(y), \quad x \in \mathbb{R}^{2} \backslash \bar{D},
$$

where $\Phi(x, y)$ is the fundamental solution to the Helmholtz equation in two dimensions and $\varphi$ is a continuous density (source) function defined over $\Gamma_{0}$. The far field pattern of the single-layer potential should be measured far field pattern $u^{(\infty)}$, which leads to the linear equation

$$
u^{(\infty)}(\widehat{x})=c \int_{\Gamma_{0}} e^{-i \kappa \hat{x} \cdot y} \varphi(y) d s(y), \quad \widehat{x} \in \mathbb{S}
$$

with some constant $c$ depending on the dimension of the space under consideration. The first step consists of solving the ill-posed linear equation (23) to calculate $\varphi$.

Then, when we consider a Dirichlet boundary condition, the shape is found in a second nonlinear step as the zero curve of the total field $\widetilde{u}(x)$. To find this zero curve, we introduce an operator $G$, which maps $\Gamma$ to the values of the approximate total field $\tilde{u}$, on $\Gamma$ such that

$$
G:\left.\Gamma \longmapsto \widetilde{u}\right|_{\Gamma}
$$

Then the problem is reduced to the solution of the following optimization problem:

$$
G(\Gamma)=0,
$$

which can be done by minimizing the defect $\|G(\Gamma)\|_{L^{2}(\Gamma)}$ in a least square sense.

Linear sampling [70-74] is based on solving the far field equation

$$
\int_{\Omega} u_{\infty}(\widehat{x} ; d) g(d) d s(d)=\frac{e^{i \pi / 4}}{\sqrt{8 \pi k}} e^{-i k \hat{x} \cdot y} \quad \hat{x} \in \mathbb{S} .
$$

Then, the density $g$ satisfies

$$
\|g(\cdot, y)\|_{L^{2}(\Omega)} \longrightarrow \infty, \quad y \longrightarrow \partial D,
$$

that is, when the source point $y$ approaches the boundary $\Gamma$. This behaviour can be used to visualize the shape of the scatterer from the knowledge of the far field pattern $u_{\infty}(\cdot, d)$ for all directions $d \in \mathbb{S}$.

We also refer to the orthogonality sampling method, which has recently been suggested in [86]. It is particularly suited to deal with multifrequency data as is naturally obtained when acoustic pulses are used to probe an object or region in space. The method has been independently suggested by Ito et al. $[87,88]$ and successfully applied as a first step in a larger inversion procedure. A convergence analysis of the method in the limit of small scatterers has recently been achieved by Griesmaier [89].

\subsection{Parameter on the Boundary Reconstruction Problems} of Acoustic Waves. The inverse problem considered in this section is finding a continuous function which is defined on the boundary of the obstacle from the knowledge of the scattered acoustic waves for a given shape in two dimensions. We discuss the methods whose aims are to reconstruct $\eta$, in the impedance boundary condition (6), and $\lambda$, in the conductive boundary condition (7). Note that conductivity function is employed to model the inhomogeneity which might exist on the boundary of an object in a more realistic way.

In [90-92], 3D obstacles with impedance boundary condition are studied for acoustic case, where in [91] electromagnetic case is included. In the same field, the papers $[64,93-$ 96] are focused on 2D geometries for the less complexity of governing numerical experiments.

In [90], the impedance reconstruction problem is solved theoretically with a technique depending on Backus and Gilbert's method which is applicable to linear moment problems. To this aim, approximate Green's functions are used to reduce the nonlinear problem to two linear moment problems. On the other hand, the study [92] is devoted for the reconstruction of impedance functions via the KirschKress and Colton-Monk decomposition methods. Furthermore, some interesting papers appeared on the impedance reconstructions, recently $[97,98]$.

The paper [93] introduced a new method for impedance reconstructions in the spirit of the Kirsch-Kress decomposition method. That is, the scattered field is represented via single-layer potential over the known boundary of the impedance cylinder $\Gamma$, instead of defining an auxiliary initially guessed curve. Then, the density function $\varphi$ is solved from the following ill-posed integral equation

$$
u_{\infty}(\hat{x})=\frac{e^{i \pi / 4}}{\sqrt{8 \pi k}} \int_{\Gamma} e^{-i k \hat{x} \cdot y} \varphi(y) d s(y), \quad \widehat{x} \in \Omega,
$$

through Tikhonov's regularization for known far field, where $\Omega$ is a unit circle. From the knowledge of the density function now the total field and the normal derivative of the total field can be computed on the boundary of the obstacle via 
jump relations [22]. Finally, $\eta$ is obtained from (6) in the least squares sense. This method is also extended for the reconstructions of the conductivity functions of the obstacles in free space [99], for the obstacles buried in penetrable cylinders $[100]$ and for a combination of a shape and conductivity function reconstruction problem [101], firstly by Yaman [6].

Moreover, $[64,95,96]$ are devoted for the shape and impedance reconstructions of $2 \mathrm{D}$ obstacles in acoustics. To do this, the hybrid method is employed by Serranho $[64,66]$. In [95], a level set algorithm is combined with boundary integral equations in acoustic case to reconstruct the shape and impedance of $2 \mathrm{D}$ obstacles from multi-illuminations, and in [96], it has been shown that the knowledge of the scattered fields corresponding to three incident waves can be used for the determination of the shape and the impedance via integral equation methods and conformal mapping techniques.

\section{Inverse Problems for Differential Equations of Elastodynamics}

3.1. Differential Equations of Elastodynamics. Let $x=$ $\left(x_{1}, x_{2}, x_{3}\right) \in \mathbb{R}^{3}$ be a $3 \mathrm{D}$ space variable, and let $t \in \mathbb{R}$ be a $1 \mathrm{D}$ time variable; let $\mathbf{u}(x, t)=\left(u_{1}(x, t), u_{2}(x, t), u_{3}(x, t)\right)$ be the displacement vector function of an inhomogeneous anisotropic elastic material characterizing by density $\rho$ and the elastic moduli $C_{i j k l}$. The density $\rho$ and elastic moduli $C_{i j k l}$ are varying functions of position $x=\left(x_{1}, x_{2}, x_{3}\right)$. Combining the properties of the strain-energy function with Hooke's law we find [102] that $C_{i j k l}$ satisfy the following property and strong convexity

$$
C_{i j k l}=C_{k l i j}=C_{l k i j}=C_{i j l k}, \quad \sum_{i=1}^{3} \sum_{j=1}^{3} \sum_{k=1}^{3} \sum_{l=1}^{3} C_{i j k l} \varepsilon_{i j} \varepsilon_{k l}>0
$$

for any nonzero $3 \times 3$ real symmetric matrix $\left(\varepsilon_{i j}\right)_{3 \times 3}$. Equations for motion in inhomogeneous anisotropic elastic materials are, in our notation (see, e.g., [102]),

$$
\rho \frac{\partial^{2} u_{i}}{\partial t^{2}}=\sum_{j=1}^{3} \sum_{k=1}^{3} \sum_{l=1}^{3} \frac{\partial}{\partial x_{j}}\left(C_{i j k l} \frac{\partial u_{k}}{\partial x_{l}}\right)+f_{i}, \quad i=1,2,3,
$$

where $f_{i}$ are components of the body forces $\mathbf{f}(x, t)=$ $\left(f_{1}(x, t), f_{2}(x, t), f_{3}(x, t)\right)$ acting per unit volume on the particle originally at position $x$ at some reference time $t$.

Let us examine

$$
\mathbf{u}(x, t)=\mathbf{U}(t-T(x)) A(x)
$$

as an approximate solution of (30) for $\mathbf{f}=0$. Near a wavefront $t=T(x)$, we assume that components of $\mathbf{U}=\left(U_{1}, U_{2}, U_{3}\right)$ are fluctuating much more rapidly than $A(x)$ or $C_{i j k l}$, and the successive derivatives $\partial \mathbf{U} / \partial t$ and $\partial^{2} \mathbf{U} / \partial t^{2}$ are fluctuating still more rapidly. Substituting (31) into (30) we find (see, e.g., [102])

$$
\left(\rho \delta_{i k}-\sum_{j=1}^{3} \sum_{l=1}^{3} \frac{\partial T}{\partial x_{j}} \frac{\partial T}{\partial x_{l}}\right) \frac{\partial^{2} U_{k}}{\partial t^{2}} A=E_{i}(\mathbf{U} A),
$$

where $E_{i}(\mathbf{U} A)$ includes merely first-order derivatives of $\mathbf{U}$, $\mathbf{U}$ itself, the elastic moduli, amplitude function $A(x)$, and gradients of these.

Thus, the left-hand side of (32) must be much smaller than $\partial^{2} \mathbf{U} / \partial t^{2}$. We conclude that the matrix of coefficients of $\partial^{2} \mathbf{U}(t-T(x)) / \partial t^{2} A(x)$ must be singular:

$$
\operatorname{det}\left(\rho \delta_{i k}-\sum_{j=1}^{3} \sum_{l=1}^{3} \frac{\partial T}{\partial x_{j}} \frac{\partial T}{\partial x_{l}}\right)=0 .
$$

This equation determines the possible wavefronts in an elastic medium, since it gives a constraint on the function $T(x)$. In an inhomogeneous isotropic medium,

$$
C_{i j k l}=\lambda \delta_{i j} \delta_{k l}+\mu\left(\delta_{i k} \delta_{j l}+\delta_{i l} \delta_{j k}\right),
$$

where $\delta_{i j}$ is the Kronecker symbol; that is, $\delta_{i j}=1$ if $i=j$ and $\delta_{i j}=0$ if $i \neq j$; moreover, $\lambda=\lambda(x), \mu=\mu(x)$ are known as the Lame functions.

In an inhomogeneous isotropic medium, the special form (34) of $C_{i j k l}$ makes it possible to get (33) as follows:

$$
\left(\nabla T \cdot \nabla T-\frac{\rho}{\lambda+2 \mu}\right)\left(\nabla T \cdot \nabla T-\frac{\rho}{\mu}\right)^{2}=0 .
$$

This is, $T(x)$ satisfies the eikonal equation

$$
|\nabla T|^{2}=\frac{1}{C_{P}^{2}(x)}
$$

or eikonal equation

$$
|\nabla T|^{2}=\frac{1}{C_{S}^{2}(x)}
$$

where $C_{P}^{2}=\sqrt{(\lambda+2 \mu) / \rho}$ is the local $P$-wave speed and $C_{S}^{2}=$ $\sqrt{\mu / \rho}$ is the local $S$-wave speed.

3.2. Inverse Kinematic Problem of Seismic. Suppose that a point source at position $x^{0} \in \mathbb{R}^{3}$ becomes active at a time chosen to be the origin, $t=0$. In homogeneous isotropic medium, wavefronts emanate from the source as ever-expanding spheres, with radius $C_{P} t$ (for $P$-waves) and $C_{S} t$ for $S$-waves, arriving at the general position $x$ at time $t=\left|x-x^{0}\right| / C_{P}$ and $t=\left|x-x^{0}\right| / C_{S}$. We introduce the function $T\left(x, x^{0}\right)$ as the travel time required for the wavefront to reach $x$ from $x^{0}$. The function $T\left(x, x^{0}\right)$ satisfies $(36)$ for $P$-waves and (37) for $S$-waves.

One of the first inverse problem, stated and studied in geophysics, was the inverse kinematic problem. The physical interpretation of this problem is the following. Let us assume that Earth is an isotropic inhomogeneous elastic medium and the measurements of the seismic waves, arising from a point source $x^{0}$ and propagating in Earth, are given for points on its surface. These measurements contain data of the travel time $T\left(x, x^{0}\right)$ of seismic waves between the point of the source $x^{0}$ and any point of the Earth's surface. The inverse kinematic problem is to find the speed of the seismic waves inside of 
Earth using the measurement data. Mathematically we can state the inverse kinematic problem as follows. Let $D$ be a domain bounded by the surface $S$, and let $T\left(x, x^{0}\right)$ be the function of the travel time required by a signal with unknown speed $C(x)>0$ to reach $x$ from $x^{0}$. Find $C(x)$ for all $x$ from $D$ if the function $T\left(x, x^{0}\right)$ is given for all points $x^{0} \in S_{1}$ and $x \in S_{2}$, where $S_{1} \subseteq S$ and $S_{2} \subseteq S$ are subsets of $S$.

Herglotz [103] and Wiechert and Zoeppritz [104] were the first who studied the inverse kinematic problem in assumptions

$$
\begin{aligned}
& C(x) \frac{1}{n(r)}, \quad r=|x|, \frac{d}{d r}(r n(r))>0, \\
& x^{0} \text { is a fixed point from } S,
\end{aligned}
$$

if $T\left(x, x^{0}\right)$ is known for any $x$ from $S$. Gerver and Markushevich [105] have showed that the condition $(d / d r)(r n(r))>$ 0 can be illiminated, but in this case the inverse kinematic problems have many solutions and the set of these solutions has been described. The first theoretical study of the inverse kinematic problem for a horizontal inhomogeneous medium has been made by Lavrenti'ev and Romanov [106]. The first result of the study of the multidimentional inverse kinematic problem in a linear approximation, when the function $C(x)$ depends on $3 \mathrm{D}$ space variable $x=\left(x_{1}, x_{2}, x_{3}\right) \in \mathbb{R}^{3}$, was obtained by Romanov [107]. The existence, uniqueness, and stability estimate theorems for the multidimensional inverse kinematic problems have been established in some classes of analytic and smooth functions by Anikonov [108], Bukhgeimm [109], and Mukhometov [110].

In a recent time, the Earth is modeled as an anisotropic elastic medium which is located in the given $3 \mathrm{D}$ domain. The wave speed is given by a symmetric positive definite matrix $G=\left(g_{i j}\right)_{3 \times 3}$, that is, a Riemannian metric in mathematical terms (see, e.g., [111]). The problem is to determine the metric in a given domain from the lengths of geodesics joining points on the boundary of the domain. The linearization of this problem leads to a problem of the integral geometry [111-113].

The regular study of the problems of finding the isotropic and anisotropic Riemannian metrics and integral geometry problems has been made in the works [111, 114-117]. The modern numerical algorithms for the computation of the inverse kinematic problems of seismic have been developed in the works $[118,119]$.

3.2.1. Inverse Problems in Ray Statements. Let us note that isotropic inhomogeneous elastic medium is completely characterized by three functions: the density $\rho(x)$ and speeds $C_{P}(x), C_{S}(x)$ of $P$ - and $S$-waves. Using the point source at position $x^{0}$ of the boundary $S$ of the given domain $D$, which becomes active at the time $t=0$, we measure the function $T\left(x, x^{0}\right)$ of the travel time required for the fronts of $P$ - and $S$-waves to reach $x$ from $x^{0}$. We use these information for solving two inverse kinematic problems for $P$ - and $S$-waves. The solutions of these problems are speeds $C_{P}(x), C_{S}(x)$. To complete the identification of unknown isotropic inhomogeneous medium we need to determine the last unknown function $\rho(x)$ after finding $C_{P}(x), C_{S}(x)$. An inverse problem to recover $\rho(x)$ in a given bounded domain $D$, containing an isotropic inhomogeneous elastic medium, has been solved by Yakhno [120-123]. In these works, the displacement fields $\mathbf{u}\left(x, x^{0}, t\right)$ have been measured for all points $x$ and $x^{0}$ running the boundary $S$ of $D$ for all times from a time interval containing the time $t=T\left(x, x^{0}\right)$ of arriving of the $P$-waves.

3.3. One-Dimensional Inverse Dynamic Problems. The vertical inhomogeneous model of Earth is one of the popular models of geophysics [102], and the inverse problems of recovering the density $\rho\left(x_{3}\right)$ and Lame functions $\mu\left(x_{3}\right)$ and $\lambda\left(x_{3}\right)$, depending on one variable $x_{3}$ and appearing in equations of elastodynamics (30) for the case of inhomogeneous isotropic elastic medium, have been studied by many authors $[1,2,7-15,21,22,24,26-28,36-46,53-55,59-$ 61, 70-73, 78-81, 85, 87-91, 97, 98, 103, 105, 108, 109, 113, $118,119,124-214]$. Because the unknown functions depend on one variable, the inverse problems of their recovering are called one-dimensional inverse problems although all differential equations of elasticity contain $3 \mathrm{D}$ space variable $x=\left(x_{1}, x_{2}, x_{3}\right)$ and 1D time variable $t$. Alekseev and Dobrinsky $[124,125]$ were the first who described the importance of one-dimensional inverse problems of elastodynamics in geophysics and studied them as problems of the recovery of smooth functions $\rho\left(x_{3}\right), \mu\left(x_{3}\right)$, and $\lambda\left(x_{3}\right)$ of one variable $x_{3}$. The uniqueness of the solutions of these inverse problems has been studied firstly by Blagoveschenskii [147] and Romanov [215] for the isotropic elastic media and then by Volkova and Romanov [216] for anisotropic elastic media. The regular study of the theory, methods, and applications of one-dimensional inverse problems for dynamical differential equations of isotropic and anisotropic elastic media has been made in works [122, 159, 170, 190, 217-221] and others. The recent development of theory, methods, and applications of one-dimensional inverse problems of dynamic elasticity can be found in the works [150, 211, 222, 223].

We note that a model of Earth as a composite medium consisting of a finite number of different elastic layers is very popular in geophysics. In this case, the one-dimensional inverse problem consists of finding $\rho\left(x_{3}\right)$ and the Lame functions $\mu\left(x_{3}\right)$ and $\lambda\left(x_{3}\right)$ as functions of one variable $x_{3}$ with piecewise constant values. The computation of solutions of this type of one-dimensional inverse problems has been studied in $[186,187,220]$. The modern theory and methods of the construction of solutions of one-dimensional inverse problems for the equations of elastodynamics in elastic composite media can be found in the works $[142,214,224-$ 228].

3.4. Multidimensional Inverse Dynamic Problems in Linear (Born) Approximation. Linearized multidimensional inverse dynamic problems (or inverse problems in the Born approximation) take an important place through all statements of multidimensional inverse problems for equations of elastodynamics. The statements of these problems have natural physical and mathematical sense. From the physical point of view, an isotropic inhomogeneous elastic body, which is 
characterized by the Lame functions $\mu_{1}(x)$ and $\lambda_{1}(x)$ and density $\rho_{1}(x)$, is included in a vertical inhomogeneous (or homogeneous) elastic medium. Let, for example, the half space $x_{3}>0$ contain this medium, and let the characteristics $\mu_{1}(x), \lambda_{1}(x)$, and $\rho_{1}(x)$ of the elastic body be unknown functions. The linearized inverse problem is to find these unknown functions if we measure the first act of scattering the displacement field on the surface $x_{3}=0$ arising from the forces located on the same surface $x_{3}=0$. From the mathematical point of view, we consider the differential equations of elastodynamics in a half space $x_{3}>0$ with boundary conditions on $x_{3}=0$. We assume that the Lame functions $\mu(x)$ and $\lambda(x)$ and density $\rho(x)$ appearing in differential equations and boundary conditions can be presented in the form

$$
\begin{aligned}
& \mu(x)=\mu_{0}\left(x_{3}\right)+\mu_{1}(x), \\
& \lambda(x)=\lambda_{0}\left(x_{3}\right)+\lambda_{1}(x), \\
& \rho(x)=\rho_{0}\left(x_{3}\right)+\rho_{1}(x),
\end{aligned}
$$

where $\mu_{0}\left(x_{3}\right), \lambda_{0}\left(x_{3}\right)$, and $\rho_{0}\left(x_{3}\right)$ are functions depending on $x_{3}$ and characterizing vertical inhomogeneous medium and $\mu_{1}(x), \lambda_{1}(x)$, and $\rho_{1}(x)$ are functions of 3D space variable $x=$ $\left(x_{1}, x_{2}, x_{3}\right)$ characterizing the elastic body which is included in the vertical inhomogeneous medium.

We assume that the displacement field $\mathbf{u}(x, t)$ is presented in the form $\mathbf{u}(x, t)=\mathbf{u}_{0}(x, t)+\mathbf{u}_{1}(x, t)$, where $\mathbf{u}_{0}(x, t)$ is the displacement field of the vertical inhomogeneous medium arising from the given forces, and $\mathbf{u}_{1}(x, t)$ is the first act of scattering $\mathbf{u}_{0}(x, t)$ on the inhomogeneous inclusion with characteristics $\mu_{1}(x), \lambda_{1}(x)$, and $\rho_{1}(x)$. The equations of elastodynamics with boundary conditions are linearized around $\mu_{0}\left(x_{3}\right), \lambda_{0}\left(x_{3}\right), \rho_{0}\left(x_{3}\right)$, and $\mathbf{u}_{0}(x, t)$. The unknown functions $\mu_{1}(x), \lambda_{1}(x)$, and $\rho_{1}(x)$ appear in inhomogeneous terms of linearized equations for $\mathbf{u}_{1}(x, t)$. We need to recover $\mu_{1}(x), \lambda_{1}(x)$, and $\rho_{1}(x)$ if we know $\mathbf{u}_{1}(x, t)$ for $x_{3}=0$ (see, e.g., [122]). The uniqueness of the solution of a linearized multidimensional inverse problem has been studied by Romanov [215]. The existence theorem and computation of a solutions of a linearized multidimensional inverse problems of elastodynamics have been obtained in the works $[122,229]$. The recovery of the function characteristics of an elastic body in linear approximation was a subject of the works $[146,154$, 202, 230]. The linearized inverse problems of determining the function characteristics of transversally isotropic elastic media from the measurements of reflected waves have been developed by Sharafutdinov [231, 232]. The linearized inverse problems for nonhomogeneous bodies have been stated and developed by Steinberg [233].

3.5. Multidimensional Inverse Dynamic Problems in the Statements of the Dirichlet-To-Neumann Map. The inverse problems of determining the elastic moduli and density as functions of the space variables in a bounded domain from observed data of the solution on the boundary (or a part of the boundary) of this domain are geophysical motivated. One important class of these problems is inverse problems for equations of elastodynamics in terms of the Dirichletto-Neumann map. The Dirichlet-to-Neumann map models surface measurements by giving the correspondence between a displacement at the boundary $S$ of the given bounded domain $D$ and surface traction

$$
\left.\sum_{j=1}^{3} \sum_{k=1}^{3} \sum_{l=1}^{3} v_{j} C_{i j k l} \frac{\partial u_{k}}{\partial x_{l}}\right|_{S \times(0, T)}, \quad i=1,2,3,
$$

where $v=\left(v_{1}, v_{2}, v_{3}\right)$ is the unit outer normal to $S,(0, T)$ is the observed time interval, and $u_{k}, k=1,2,3$ are components of the displacement vector function $\mathbf{u}(x, t)$ satisfying (30). The details of the use of the Dirichlet-to-Neumann map in modeling surface measurements in inverse problems can be found in [234]. The inverse problems in the Dirichlet-toNeumann map statements are successfully applied to study the unique determination of the solutions of the inverse problems of elasticity as in the static isotropic and anisotropic cases [235-239] as well as in dynamic case [240].

3.6. Carleman Estimates and Uniqueness of Solutions for Multidimensional Inverse Dynamic Problems. For the study of the inverse problems for the scalar partial differential equations with a finite number of observation, Bukhgeim and Klibanov [160] proposed a remarkable method based on a Carleman estimate. Later, the Carleman estimate method has been generalized to study the uniqueness and stability estimate of the solutions of the inverse problems for equations of elastodynamics by Isakov [208], Ikehata et al. [203], Eller et al. [182], Imanuvilov et al. [204], Imanuvilov and Yamamoto $[205,206]$, and Lin and Nakamura [241].

In the papers $[203-206,208]$ the authors assume some geometric constraints on the surface under observation for proving the uniqueness and stability of the solutions of the multidimensional inverse problems using the Carleman estimates. Later, the stability estimate theorem for solutions of a multidimensional inverse problem for equations of elastodynamics has been proven for an arbitrary subboundary by Bellassoued et al. [143].

\section{Inversion Based Applications}

In the following, we present a general overview on some inversion-based application areas. We then give more details about particular selected applications in the subsequent sections. Further, different applications whose details are skipped in this section such as remote sensing, nuclear science, and geophysics can be found in [164, 184, 242-247].

Underwater Acoustics and Traveltime Tomography. Inverse problems related to underwater acoustics are a critical research area due to their wide range of important practical applications. Solutions of such problems can provide a heat distribution of seawater, an ocean depth at a point, sediment properties of a seabed, locations and/or shapes of submerged objects (mines, submarines, sunken wrecked ships buried to the seafloor, etc.), positions of underwater cables (communication, gas, petroleum, waste, etc.), profiles of seamounts, and a layered seabed for seismic applications 
or paths for current flows and migrations of sea animals [21]. In principle, all these applications can roughly be classified into two groups of problems such as remote sensing of passive marine environments and localization/characterization of acoustic sources which are in the sea or buried in the seafloor [171]. For the solution of these problems, one can use the speed of acoustic wave as a tool which is obtained from traveling time of an acoustic wave between two certain points or the amplitude and/or phase information of acoustic waves reflected/scattered from a target(s). In water-type medium, propagating acoustic waves are collected at a selected number of separate hydrophones to obtain measured field data.

Generally, reconstructions of desired parameters from the knowledge of scattered acoustic waves lead to nonlinear and ill-posed inverse problems. Therefore, it is always a complicated issue to find unique and stable solutions, and one has to apply some additional techniques, that is, regularizations, for the proper treatment of the ill-posedness. More specifically, researchers have been applying the methods of Ray's theory, modal travel time/phase inversions, boundary integral equation approaches, Rayleigh's hypothesis, linear sampling, complete family method, convex scattering support theory, and so forth, in underwater acoustics [21, 71, 153, $165,171,189,248-252]$.

Nondestructive Testing. The inspection of an object without touching or without changing its characteristic properties is a general definition of nondestructive testing (NDT) in the literature. In practice, NDT is commonly performed by following visual, penetrant, magnetic particle, radiographic, thermal infrared, Eddy's current, and ultrasonic type testing procedures for the identifications of cracks, shapes of surface discontinuities, or corrosion damages in power plants, rails, pipelines, bridges, buildings, airplanes, railroad tank cars, and so forth, $[196,253]$. The main idea of these methods, which actually shows the conceptual relation between inverse problems and NDT applications, is the evaluation of the responses obtained from the interaction of a test object with a particular effect, that is, electromagnetic/acoustic field, gamma or X-radiation, fluorescent dye, and so forth. Therefore, approaches which are used for inverse problems, that is, synthetic aperture focussing technique (SAFT, $[180,254]$ ), diffraction tomography (DT, [177-179, 255-257]), multiple signal classification (MUSIC, [129, 155, 167, 176, 198]), linear sampling method (LSM, [70-74]), factorization [75, 76, 258], point source $[23,68,69]$, no response test [82-84], and so forth, are also employed for NDT problems [259].

More specifically, SAFT is an algorithm which uses the collection of echo signals over a specific aperture to obtain a reconstruction by performing time shifting and superposition of adjacent signals. DT is based on a linear solution of the wave equation which can be obtained via the Born or Rytov approximations. Here, the linearization approaches define mainly the success and the solution space of the inverse problem. MUSIC was initially employed as a direct imaging algorithm to obtain locations of point scatterers [176] and extended to find also the geometry of targets [198]. The method employs the eigenvalue structure of time-reversal matrix which is obtained from measured data at different receiver antennas. The main idea of linear sampling method is to find an indicator function such that its value provides whether an arbitrarily tested space coordinate lies inside or outside of the object.

Biomedical Imaging Techniques, Tomography, and EIT. Nowadays medical imaging, which can be considered as one of the most developed area of inverse problems in practice, provides high-resolution reconstructions in the order of millimeters. In the last decays, the main effort is given for the implementation of harmless, fast, cheap, robust, and reliable techniques to use in practice for obtaining high-resolution images in real time. In the similar direction, early studies of bioimaging started with the reconstructions of $2 \mathrm{D}$ images of human body parts via the inverse Radon transform [145, $162,260]$ of measured $\mathrm{X}$-rays which were attenuated inside the body $[168,261]$. Afterwards, 3D images were assembled via computed tomography (CT-scan) from a series of $\mathrm{X}$ ray data measured on different planes (sinograms) in $2 \mathrm{D}$ $[144,199,212,262,263]$. Even though satisfactory results were obtained with the X-ray radiology especially for the bone structures $[131,264]$ the method was found not sufficiently efficient due to attenuation characteristics of X-rays and harm risks of using ionized radiation on humans. On the other hand, electrical impedance tomography (EIT), after its main idea and formulation were introduced by Calderon in 1980 [163] and D. Isaacson and E. L. Isaacson [207], has gained high interest both from theoretical and physical point of view. In principle, in EIT low-frequency electrical currents are applied to the body part under investigation. Then electrical properties of body tissues are computed from the measurements of electric currents and voltage at the boundary $[126,136,151$, $152,156,166,197,265-268]$. EIT is successfully applied for diagnosis of breast cancer, monitoring brain and gastrointestinal functions, detection of blood clots in the lungs, and so forth [138]. Furthermore, electroencephalography (EEG) and magnetoencephalography (MEG) are used for passive monitoring of neuron activities in the brain from the weak electric or magnetic fields, respectively, [130, 137, 169, 191, 269, 270].

MRI, PET, and SPECT. A different approach which is based on using properties of subatomic particles with the connection to electromagnetism opened a new area for obtaining high spatial resolutions in bioimaging, for example, magnetic resonance imaging (MRI), positron emission tomography (PET), and single-photon emission computed tomography (SPECT). In MRI, the patient stays in a tunnel under a strong magnetic field typically $0.2-1.5 \mathrm{~T}$. This large static magnetic field aligns protons of many atoms either parallel or antiparallel existing in the body. In the meantime, weak radio frequency fields are applied systematically to the patient for altering the alignment of the magnetization. As a result of this procedure, rotating magnetic fields induce a voltage at the receiver coils of the magnet which is used to reconstruct the image of the scanning area [131, 192, 271-274]. In PET and in SPECT, chosen molecules are labeled with radioactive atoms having short half-life and injected to the patients' bloodstream at very low concentrations in order not to violate radiation exposure limits. For PET, labeled atoms are chosen 
to emit positrons, and for SPECT, they are chosen to emit photons when they are decaying. Gamma rays, which occur when an electron and an emitted positron annihilate in PET, and photons which are released in SPECT can be visualized out of body by using scintigraphic detectors for clinical applications of oncology, cardiology, pharmacology, and so forth [127, 175, 275-282].

MW/Ultrasound Tomography, Optical Imaging, and Cognition. Another group of techniques such as microwave tomography, ultrasound, and optical imaging, which are commonly used for solutions of inverse problems in different areas, are also applied to biomedical applications especially for investigating soft body tissues $[135,148,149,185,210$, 283-289]. For instance, optical tomography is used for the detection of cancerous cells in breast and brain. Acoustic waves are employed for the imaging of liver, kidney, fetus in pregnant women, and so forth, and microwaves are used in mammography and diagnosis of leukemia [131, 144].

Data Assimilation. Over the past two decades, it has became feasible to simulate atmospheric and geophysical processes from large-scale atmospheric flow down to convective processes on a kilometer scale. This led to the need to determine initial conditions for simulations and forecasts from a collection of diverse direct and indirect measurements, and the field of data assimilation arose.

Inverse Problems in Biological and Environmental Applications. Inverse problems are of growing importance in many parts of medicine or biology as well as in environmental applications. Here, we will provide a brief introduction into two areas, first into recent results of neural field theory and second into the basic setup of data assimilation as it is, for example, used in numerical weather prediction or for climate projections, which usually incorporates various inverse problems.

4.1. Inverse Neural Field Theory. Neural activity is often modelled by the activity potential $u(x)$ in some domain $D$. The activity potential $u$ satisfies some integro-differential equations, which in its simplest form has been suggested by Wilson and Cowan [290, 291] and Amari [128]:

$$
\begin{array}{r}
\tau \frac{\partial u(x, t)}{\partial t}=-u(x, t)+\int_{D} w(x, y) f(u(y, t)) d y \\
x \in D, \quad t \geq 0
\end{array}
$$

where $f$ is some nonlinear function which usually is used to model the firing rate of neurons in dependence of their current action potential and $\tau>0$ is a constant. The kernel $w(x, y)$ models the strength of the influence of an excitation at point $y$ to the neural field at point $x$. The first term of the right-hand side of (41) generates the decay of the activity potential $u$ in the absence of excitation or inhibition. Neural fields have been widely studied in recent years, with applications to a wide range of medical phenomena starting from electroencephalogram (EEG) and magnetoencephalogram (MEG) rhythms to robotic behaviour-for an extensive literature list we refer to [172].

Neural network, that is, the discrete version of neural fields, has attracted strong interest over many years and is a standard tool today in many applied parts of science. Neural networks have also been used to solve inverse problems, compare, for example, [292, 293] for some recent papers and further citations. However, here we want to look into inverse problems which arise in the modelling of neural activities themselves. Training of neural networks or neural fields is, in general, an ill-posed inverse problem, as we will see in due course.

Inverse neural field theory investigates the construction of connectivity kernels $w$ given some dynamics $u(x, t)$ for $x \in D$ and some time intervals $t \in[0, T]$. This so-called full-field neural inverse problem is linear and ill-posed in the sense of Hadamard, as can be readily seen by the following transform. We define

$$
\begin{gathered}
\psi(x, t):=\tau \frac{\partial u(x, t)}{\partial t}+u(x, t), \quad x \in D, t \in[0, T], \\
\varphi(y, t):=f(u(y, t)), \quad y \in D, t \in[0, T] .
\end{gathered}
$$

If we further define the operator

$$
(W \varphi)(x):=\int_{D} w(x, y) \varphi(y) d y, \quad x \in D,
$$

equation (41) obtains the form

$$
\psi(\cdot, t)=W \varphi(\cdot, t), \quad t \in[0, T] .
$$

The task is to find the operator $W$ given the family of states $\varphi(\cdot, t)$ and corresponding images $\psi(\cdot, t)$ for $t \in[0, T]$. Changing our perspective slightly, introducing the operator

$$
(K g)(t):=\int_{D} \varphi(y, t) g(y) d y, \quad t \in[0, T],
$$

we transform (41) or (44) into

$$
\psi(x, t)=K w(x, \cdot)(t), \quad t \in[0, T]
$$

for each fixed $x \in D$. For each $x \in D$, (46) is an integral equation of the first kind for the function $w(x, \cdot)$. Usually we need some smoothness of the potential $u$ with respect to its arguments $y \in D$ and $t \in[0, T]$. In this case, the operator $K$ is an integral operator with continuous kernel, which is known to be compact in either $L^{2}(D)$ or $C(D)$ (cf. [22]) and cannot have a bounded inverse. Thus, the inverse neural field problem is ill-posed.

The particular form (46) provides a basis for kernel construction, that is, for the solution of the neural field problem. We may apply spectral regularization schemes as described in $[1,22,85]$, for example, Tikhonov regularization in a similar sense of (21)

$$
R_{\alpha}:=\left(\alpha I+K^{*} K\right)^{-1} K^{*}, \quad \alpha>0,
$$


with regularization parameter $\alpha>0$ as regularized inverse to calculate $w(x, \cdot)$ from the knowledge of $\psi$ and $\varphi$. For smooth dynamics, the problem is exponentially ill-posed. We refer to $[139-141,294]$ for the analysis and many examples for the inverse neural field problem. The problem of the ill-posedness of the neural inverse problem is addressed in [295], where a dimensional reduction approach is suggested to decompose the large and strongly ill-posed full problem into more stable individual tasks.

4.2. Data Assimilation with an Application to Numerical Weather Prediction (NWP). In inverse problems, we are usually given measured data, and the task is to gain insight into some unknown parameter functions insight of an inaccessible body or area of space or to reconstruct the shape of scatterers or inclusions, but often, the quantities under reconstruction are not static but dynamic and change over time. Then, the inverse task is not only carried out once but the reconstruction is repeated over time with some cycling frequency given by a time interval $\delta T$.

Let $f_{k} \in Y$ for $k \in \mathbb{N}$ be an element of a Hilbert space $Y$ representing our measurement data. The task is to reconstruct some state $x_{k} \in X$ in a Hilbert space $X$, where the measurement is described by an observation operator $H_{k}$ : $X \rightarrow Y$. An underlying dynamical system is given by some operator $M_{k}: X \rightarrow X$, mapping the state $x_{k}$ at time $t_{k}$ onto the state $x_{k+1}$ at time $t_{k+1}$. With the reconstruction $x_{k-1}^{(a)}$ from the previous time step, we can calculate a state $x_{k}^{(b)}$ at time $t_{k}$, which serves as a priori knowledge for the current reconstruction when data $f_{k}$ are given.

The goal of data assimilation in its simplest form is to employ measurement data $f_{k}$, the operator $H_{k}$, and the background state $x_{k}$ to calculate a so-called analysis $x_{k}^{(a)}$, which is the best possible estimate of the true state $x_{k}^{\text {(true) }}$ of the dynamical system at time $t_{k}$ under the given assumptions.

Data assimilation is needed as soon as dynamic situations are studied. For example, the reconstruction of a current density from magnetic fields leads to static magnetic tomography, as, for example, solved in [193-195, 296], but when the underlying currents show a dynamic behaviour, we need to carry out dynamic magnetic tomography [297, 298], which is a data assimilation problem.

The data assimilation scheme can also use measurements from an interval of time steps, for example, a window consisting of $L$ time steps with data $f_{k-L}, f_{k-L+1}, \ldots, f_{k}$. In this case, the task is to fit the trajectory given by

$$
\begin{gathered}
H x_{k-L}, \\
H x_{k-L+1}=H M_{k-L} x_{k-L}, \\
H x_{k-L+2}=H M_{k-L+1} M_{k-L} x_{k-L}, \ldots, \\
H x_{k}=H M_{k-1} M_{k-2} \cdots M_{k-L} x_{k-L}
\end{gathered}
$$

to the measured data $f_{k-L}, \ldots, f_{k}$ given the a priori information $x_{k-L}^{(b)}$.
Let us denote the analysis or reconstruction, respectively, for this window $\left[t_{k-L}, t_{k}\right]$ at time $t_{k-L}$ by $\tilde{x}_{k-L}^{(a, L)}$. Then, it leads to a state estimate at time $t_{k}$ by

$$
x_{k}^{(a)}:=M_{k-1} M_{k-2} \cdots M_{k-L} \tilde{x}_{k-L}^{(a, L)}, \quad k \in \mathbb{N} .
$$

An algorithm, which calculates $\tilde{x}_{k-L}^{(a)}$ at the beginning of the window $\left[t_{k-L}, t_{k}\right]$, is usually denoted as smoother. The calculation of $x_{k}^{(a)}$ at the end of the interval is called a filter. There is a wide range of literature about data assimilation, in particular from the perspective of filtering. We refer to $[133,134,173,209,213,299-304]$ for introduction and further details, in particular in atmospherical sciences.

We can reformulate the data assimilation task as a minimization, here written in its usual finite-dimensional version. If we carry out minimization independently at every time $t_{k}$, the scheme is known as three-dimensional variational assimilation (3dVar) and minimizes

$$
J\left(x_{k}\right):=\left\|x_{k}-x_{k}^{(b)}\right\|_{B^{-1}}^{2}+\left\|f_{k}-H x_{k}\right\|_{R^{-1}}^{2}
$$

where $B$ and $R$ are weight matrices in $X$ and $Y$, respectively. In a stochastic framework, $B$ is the covariance matrix of background distribution of states in $X$ and $R$ is the covariance matrix of the data error in $Y$.

The 3dVar algorithm is basically a version of the wellknown Tikhonov regularization in a Hilbert space where weighted norms are used. Also, it can be seen as a version of the Bayes approach for a Gaussian densities; compare [209] for an introduction and [188] for a recent survey.

When a window of measurements is used, the corresponding minimization problem is given by

$$
J\left(x_{k-L}\right)=\left\|x_{k-L}-x_{k-L}^{(b)}\right\|_{B^{-1}}^{2}+\sum_{\ell=1}^{L}\left\|f_{k-L+\ell}-H x_{k-L+\ell}\right\|_{R^{-1}}^{2},
$$

where $x_{k-L+\ell}$ is given by (48). The minimization of (51) is denoted as four-dimensional variational assimilation (4dVar). The 4dVar algorithm is known to be expensive both in terms of computing time and programming effort, since usually the adjoint tangent linear model of the dynamics needs to be calculated and implemented, but 4dVar has turned out to be the algorithm which provides best scores in the area of numerical weather forecasting (NWP); compare the scores which are available from the European Centre for Medium Range Weather Forecast (ECMWF) [174].

For linear systems the minimization of the $4 \mathrm{dVar}$ functional (51) can be carried out iteratively with individual steps as in (50). The key difference to 3dVar is a dynamic update of the covariance matrix $B$ in every time step. The method is the well-known Kalman Filter (KF) [213]. The Kalman filter is often studied in a stochastic framework, for example, [209, 304], where it calculates the maximum probability estimator (MAP) for the Bayes formula in a Gaussian framework and when the prior density has a covariance matrix $B$ and mean $x_{k}^{(b)}$. A simple algebraic equivalence proof of the Kalman smoother/filter to 4 dVar is presented, for example, 
in [188]. We need to remark that for large-scale systems, the update of the $B$ matrix in the Kalman filter is not feasible, which has led to the development of ensemble and particle methods.

Ensemble or particle methods which are well-known in stochastic estimation since around 1980 (Markov Chain Monte Carlo methods, MCMC) have become very popular in the area of data assimilation. The basic idea here is to estimate the covariance matrix $B$ by a stochastic covariance estimator, based on a set of ensembles or particles. Then, an assimilation step as in (50) is carried out. The method is known as ensemble Kalman filter (EnKF). Here, a particle or ensemble member, respectively, is basically a state $x_{k-1}$, and its trajectory $x_{k}=M_{k-1} x_{k-1}$. When a distribution of states is given, we can calculate mean and covariance by standard tools. The propagation of these states through time using the dynamical system $M$ leads to an estimate of mean and covariance of the propagated distribution at a later point in time.

The estimation of the covariance matrix $B$ by an ensemble with 40,100 , or 200 members in a high-dimensional space leads to spurious covariances, which make the ensemble approach useless for such systems. As a consequence, ensemble data assimilation systems have employed localization [132, $181,200,305,306]$. Here, calculations are carried out in a local region only, not the global space $[307,308]$. The local ensemble Kalman filter (LEKF) is studied in [306], and its computationally more efficient version has been developed by Hunt et al. [201], the local ensemble transformed Kalman filter (LETKF).

More recently, there has been increasing interest in the study of stability of data assimilation algorithms over time, in particular when ill-posed operators are involved in the observation process; compare $[157,158,309,310]$. The key object here is to study the mapping of the data sequence onto the analysis sequence

$$
\left(f_{k}\right)_{k \in \mathbb{N}} \longmapsto\left(x_{k}^{(a)}\right)_{k \in \mathbb{N}^{*}}
$$

Stability-estimating $\left\|x_{k}^{(a)}\right\|$ for $k \rightarrow \infty$-has been derived under particular conditions, leading to tools to control the stability over time by scaling the background covariance matrix $B$ (or alternatively the data covariance matrix $R$ ) that have been developed [311].

\section{Conclusions}

In this paper, inverse problems are reviewed starting with a historical perspective. Mathematical backgrounds for the fundamental inverse problems in acoustics, electromagnetics, and elastics are given. The main ideas of many algorithms whose aims to find locations, shapes, and boundaryparameters of obstacles are introduced by studying two dimensional acoustic cases for the sake of simplicity of the theoretical investigations.

Furthermore, the results of the study of the inverse problems for the time-dependent differential equations of isotropic and anisotropic elasticity are presented chronologically from one-dimensional kinematic and dynamic inverse problems with essentially over-determined data up to multidimensional inverse problems with a finite number of observations. The physical and geophysical interpretations are supplied for almost all reviewed works.

We note that the study of one-dimensional and linearized multidimensional inverse problems for equations of elastodynamics contains the theory (existence, uniqueness, and stability theorems) and computational methods of finding solutions. The study of the multidimensional dynamic problems for the differential equations of elasticity contains mainly the investigations on the uniqueness of the solutions as well as stability to the variation of data. At the same time, computational methods of solving the multidimensional inverse dynamic problems are not as widely developed. The theory and methods of solving the direct and inverse problems for classical equations of elastodynamics have multiple applications in geophysics and engineering.

In the last part of the paper, practical applications of inverse problems are summarized. Moreover, a brief introduction into recent results of neural field theory is provided since inverse problems arising in cognitive sciences have become popular in an interdisciplinary community of cognitive neuroscience. As a final application basic setup of data assimilation as it is, for example, used in numerical weather prediction or for climate projections is investigated in details.

\section{References}

[1] C. W. Groetsch, Inverse Problems in the Mathematical Sciences, Vieweg Mathematics for Scientists and Engineers, Friedrich Vieweg \& Sohn, Braunschweig, Germany, 1993.

[2] J. Hadamard, Lectures on Cauchys Problem in Linear Partial Differential Equations, Yale University Press, New Haven, Conn, USA, 1923.

[3] A. Kirsch, An Introduction to the Mathematical Theory of Inverse Problems, vol. 120 of Applied Mathematical Sciences, Springer, New York, NY, USA, 1996.

[4] R. G. Newton, "Inverse problems in physics," SIAM Review, vol. 12, pp. 346-356, 1970.

[5] R. L. Parker, "Understanding inverse theory," Annual Review of Earth and Planetary Sciences, vol. 5, pp. 35-64, 1977.

[6] F. Yaman, Inverse scattering problems for the objects buried in penetrable cylinders [Ph.D. thesis], Istanbul Technical University, 2009.

[7] W. M. Boerner, A. K. Jordan, and I. W. Kay, "Introduction to the special issue on inverse methods in electromagnetics," IEEE Transactions on Antennas and Propagation, vol. 29, no. 2, pp. 185-189, 1981.

[8] F. Cakoni, "Recent developments in the qualitative approach to inverse electromagnetic scattering theory," Journal of Computational and Applied Mathematics, vol. 204, no. 2, pp. 242-255, 2007.

[9] F. Cakoni and D. Colton, Qualitative Methods in Inverse Scattering Theory, Interaction of Mechanics and Mathematics, Springer, Berlin, Germany, 2006.

[10] D. Colton, J. Coyle, and P. Monk, "Recent developments in inverse acoustic scattering theory," SIAM Review, vol. 42, no. 3, pp. 369-414, 2000.

[11] D. Colton, "Inverse acoustic and electromagnetic scattering theory," in Inside Out: Inverse Problems and Applications, vol. 
47 of Mathematical Sciences Research Institute Publications, pp. 67-110, Cambridge University Press, Cambridge, UK, 2003.

[12] D. Colton and R. Kress, "Using fundamental solutions in inverse scattering," Inverse Problems, vol. 22, no. 3, pp. R49-R66, 2006.

[13] C. W. Groetsch, "Integral equations of the first kind, inverse problems and regularization: a crash course," Journal of Physics, vol. 73, no. 1, Article ID 012001, pp. 1-32, 2007.

[14] A. Hasanov, "Some new classes of inverse coefficient problems in non-linear mechanics and computational material science," International Journal of Non-Linear Mechanics, vol. 46, pp. 667684, 2011.

[15] S. I. Kabanikhin, "Definitions and examples of inverse and illposed problems," Journal of Inverse and Ill-Posed Problems, vol. 16 , no. 4, pp. 317-357, 2008.

[16] R. Kress, "Numerical methods in inverse obstacle scattering," in Inverse Problems of Wave Propagation and Diffraction, vol. 486 of Lecture Notes in Physics, pp. 93-106, Springer, Berlin, Germany, 1997.

[17] R. Kress, "Integral equation methods in inverse obstacle scattering," The ANZIAM Journal, vol. 42, no. 1, pp. 65-78, 2000.

[18] R. Kress, "Uniqueness and numerical methods in inverse obstacle scattering," Journal of Physics, vol. 73, no. 1, Article ID 012003, pp. 1-16, 2007.

[19] R. Potthast, "A survey on sampling and probe methods for inverse problems," Inverse Problems, vol. 22, no. 2, pp. R1-R47, 2006.

[20] O. Scherzer, Ed., Handbook of Mathematical Methods in Imaging, Springer, New York, NY, USA, 2010.

[21] J. L. Buchanan, R. P. Gilbert, A. Wirgin, and Y. S. Xu, Marine Acoustics: Direct and Inverse Problems, SIAM Society for Industrial and Applied Mathematics, Philadelphia, Pa, USA, 2004.

[22] D. Colton and R. Kress, Inverse Acoustic and Electromagnetic Scattering Theory, vol. 93 of Applied Mathematical Sciences, Springer, Berlin, Germany, 2nd edition, 1998.

[23] R. Potthast, Point Sources and Multipoles in Inverse Scattering Theory, vol. 427 of Chapman \& Hall/CRC Research Notes in Mathematics, Chapman \& Hall/CRC, Boca Raton, Fla, USA, 2001.

[24] M. Hanke, A. Neubauer, and O. Scherzer, "A convergence analysis of the Landweber iteration for nonlinear ill-posed problems," Numerische Mathematik, vol. 72, no. 1, pp. 21-37, 1995.

[25] R. Kress, Linear Integral Equations, vol. 82 of Applied Mathematical Sciences, Springer, New York, NY, USA, 2nd edition, 1999.

[26] F. Hettlich, "An iterative method for the inverse scattering problem from sound-hard obstacles," in Proceedings of the International Council for Industrial and Applied Mathematics (ICIAM '95), O. Mahrenholtz and R. Mennicken, Eds., vol. 2, Akademie, Berlin, Germany, 1995.

[27] T. Hohage, "Logarithmic convergence rates of the iteratively regularized Gauss-Newton method for an inverse potential and an inverse scattering problem," Inverse Problems, vol. 13, no. 5, pp. 1279-1299, 1997.

[28] T. Hohage, "Convergence rates of a regularized Newton method in sound-hard inverse scattering," SIAM Journal on Numerical Analysis, vol. 36, no. 1, pp. 125-142, 1998.

[29] A. Kirsch, "The domain derivative and two applications in inverse scattering theory," Inverse Problems, vol. 9, no. 1, pp. 8196, 1993.

[30] R. Kress, "A Newton method in inverse obstacle scattering," in Inverse Problems in Engineering Mechanics, H. D. Bui and M.
Tanaka, Eds., pp. 425-432, Balkema, Rotterdam, The Netherlands, 1994.

[31] R. Kress, "Integral equation methods in inverse obstacle scattering," Engineering Analysis with Boundary Elements, vol. 15, no. 2, pp. 171-179, 1995.

[32] R. Kress, "Integral equation methods in inverse acoustic and electromagnetic scattering," in Boundary Integral Formulations for Inverse Analysis, D. B. Ingham and L. C. Wrobel, Eds., Advances in Boundary Elements Series, pp. 67-92, Computational Mechanics, Southampton, UK, 1997.

[33] L. Mönch, "A Newton method for solving the inverse scattering problem for a sound-hard obstacle," Inverse Problems, vol. 12, no. 3, pp. 309-323, 1996.

[34] W. Tobocman, "Inverse acoustic wave scattering in two dimensions from impenetrable targets," Inverse Problems, vol. 5, no. 6, pp. 1131-1144, 1989.

[35] S. L. Wang and Y. M. Chen, "An efficient numerical method for exterior and interior inverse problems of Helmholtz equation," Wave Motion, vol. 13, no. 4, pp. 387-399, 1991.

[36] C. Farhat, R. Tezaur, and R. Djellouli, "On the solution of threedimensional inverse obstacle acoustic scattering problems by a regularized Newton method," Inverse Problems, vol. 18, no. 5, pp. 1229-1246, 2002.

[37] H. Harbrecht and T. Hohage, "Fast methods for threedimensional inverse obstacle scattering problems," Journal of Integral Equations and Applications, vol. 19, no. 3, pp. 237-260, 2007.

[38] T. Hohage, Iterative methods in inverse obstacle scattering: regularization theory of linear and nonlinear exponentially illposed problems [Dissertation], Linz, Austria, 1999.

[39] O. Ivanyshyn, R. Kress, and P. Serranho, "Huygens' principle and iterative methods in inverse obstacle scattering," Advances in Computational Mathematics, vol. 33, no. 4, pp. 413-429, 2010.

[40] O. Ivanyshyn, "Shape reconstruction of acoustic obstacles from the modulus of the far field pattern," Inverse Problems and Imaging, vol. 1, no. 4, pp. 609-622, 2007.

[41] O. Ivanyshyn, Nonlinear boundary integral equations in inverse scattering [Ph.D. thesis], University of Gottingen, 2007.

[42] O. Ivanyshyn and R. Kress, "Identification of sound-soft 3D obstacles from phaseless data," Inverse Problems and Imaging, vol. 4, no. 1, pp. 131-149, 2010.

[43] O. Ivanyshyn and T. Johansson, "Nonlinear integral equation methods for the reconstruction of an acoustically sound-soft obstacle," Journal of Integral Equations and Applications, vol. 19, no. 3, pp. 289-308, 2007.

[44] O. Ivanyshyn and R. Kress, "Nonlinear integral equations in inverse obstacle scattering," in Mathematical Methods in Scattering Theory and Biomedical Engineering, D. I. Fotiatis and C. V. Massalas, Eds., pp. 39-50, World Scientific, Hackensack, NJ, USA, 2006.

[45] O. Ivanyshyn and R. Kress, "Nonlinear integral equations for solving inverse boundary value problems for inclusions and cracks," Journal of Integral Equations and Applications, vol. 18, no. 1, pp. 13-38, 2006.

[46] T. Johansson and B. D. Sleeman, "Reconstruction of an acoustically sound-soft obstacle from one incident field and the farfield pattern," IMA Journal of Applied Mathematics, vol. 72, no. 1, pp. 96-112, 2007.

[47] R. Kress and W. Rundell, "A quasi-Newton method in inverse obstacle scattering," Inverse Problems, vol. 10, no. 5, pp. 11451157, 1994. 
[48] R. Kress and W. Rundell, "Inverse obstacle scattering using reduced data," SIAM Journal on Applied Mathematics, vol. 59, no. 2, pp. 442-454, 1999.

[49] R. Kress and W. Rundell, "Nonlinear integral equations and the iterative solution for an inverse boundary value problem," Inverse Problems, vol. 21, no. 4, pp. 1207-1223, 2005.

[50] R. Potthast, "On the convergence of a new Newton-type method in inverse scattering," Inverse Problems, vol. 17, no. 5, pp. 14191434, 2001.

[51] R. D. Murch, D. G. H. Tan, and D. J. N. Wall, "NewtonKantorovich method applied to two-dimensional inverse scattering for an exterior Helmholtz problem," Inverse Problems, vol. 4, no. 4, pp. 1117-1128, 1988.

[52] A. Roger, "Newton-Kantorovitch algorithm applied to an electromagnetic inverse problem," IEEE Transactions on Antennas and Propagation, vol. 29, no. 2, pp. 232-238, 1981.

[53] D. Colton and P. Monk, "A novel method for solving the inverse scattering problem for time-harmonic acoustic waves in the resonance region. II," SIAM Journal on Applied Mathematics, vol. 46, no. 3, pp. 506-523, 1986.

[54] D. Colton and P. Monk, "A novel method for solving the inverse scattering problem for time-harmonic acoustic waves in the resonance region," SIAM Journal on Applied Mathematics, vol. 45, no. 6, pp. 1039-1053, 1985.

[55] D. Colton and P. Monk, "The numerical solution of the threedimensional inverse scattering problem for time harmonic acoustic waves," Society for Industrial and Applied Mathematics, vol. 8, no. 3, pp. 278-291, 1987.

[56] A. Kirsch and R. Kress, "On an integral equation of the first kind in inverse acoustic scattering," in Inverse Problems, J. R. Cannon and U. Hornung, Eds., vol. 77 of Internationale Schriftenreihe zur Numerischen Mathematik, pp. 93-102, Birkhäuser, Basel, Switzerland, 1986.

[57] A. Kirsch and R. Kress, "A numerical method for an inverse scattering problem," in Inverse and Ill-Posed Problems, H. W. Engl and C. W. Groetsch, Eds., vol. 4 of Notes and Reports in Mathematics in Science and Engineering, pp. 279-289, Academic Press, Boston, Mass, USA, 1987.

[58] A. Kirsch and R. Kress, "An optimization method in inverse acoustic scattering," in Boundary Elements IX, Fluid Flow and Potential Applications, C. A. Brebbia, W. L. Wendland, and G. Kuhn, Eds., vol. 3, pp. 3-18, Computational Mechanics, Southampton, UK, 1987.

[59] T. S. Angell, R. E. Kleinman, and G. F. Roach, "An inverse transmission problem for the Helmholtz equation," Inverse Problems, vol. 3, no. 2, pp. 149-180, 1987.

[60] T. S. Angell, R. E. Kleinman, B. Kok, and G. F. Roach, "A constructive method for identification of an impenetrable scatterer," Wave Motion, vol. 11, no. 2, pp. 185-200, 1989.

[61] D. S. Jones and X. Q. Mao, "The inverse problem in hard acoustic scattering," Inverse Problems, vol. 5, no. 5, pp. 731-748, 1989.

[62] R. Kress, "Newton's method for inverse obstacle scattering meets the method of least squares," Inverse Problems, vol. 19, no. 6, pp. S91-S104, 2003, Special section on imaging.

[63] R. Kress and P. Serranho, "A hybrid method for sound-hard obstacle reconstruction," Journal of Computational and Applied Mathematics, vol. 204, no. 2, pp. 418-427, 2007.

[64] P. Serranho, "A hybrid method for inverse scattering for shape and impedance," Inverse Problems, vol. 22, no. 2, pp. 663-680, 2006.
[65] P. Serranho, "A hybrid method for inverse scattering for soundsoft obstacles in $R^{3}$," Inverse Problems and Imaging, vol. 1, no. 4, pp. 691-712, 2007.

[66] P. Serranho, A hybrid method for inverse obstacle scattering problems [Ph.D. thesis], University of Gottingen, 2007.

[67] F. Yaman, "Location and shape reconstructions of sound-soft obstacles buried in penetrable cylinders," Inverse Problems, vol. 25, no. 6, Article ID 065005, 17 pages, 2009.

[68] R. Potthast, "A fast new method to solve inverse scattering problems," Inverse Problems, vol. 12, no. 5, pp. 731-742, 1996.

[69] R. Potthast, "A point source method for inverse acoustic and electromagnetic obstacle scattering problems," IMA Journal of Applied Mathematics, vol. 61, no. 2, pp. 119-140, 1998.

[70] T. Arens, "Why linear sampling works," Inverse Problems, vol. 20, no. 1, pp. 163-173, 2004.

[71] D. Colton and A. Kirsch, "A simple method for solving inverse scattering problems in the resonance region," Inverse Problems, vol. 12, no. 4, pp. 383-393, 1996.

[72] D. Colton, M. Piana, and R. Potthast, "A simple method using Morozov's discrepancy principle for solving inverse scattering problems," Inverse Problems, vol. 13, no. 6, pp. 1477-1493, 1997.

[73] D. Colton and P. Monk, "A linear sampling method for the detection of leukemia using microwaves," SIAM Journal on Applied Mathematics, vol. 58, no. 3, pp. 926-941, 1998.

[74] R. Kress and L. Kühn, "Linear sampling methods for inverse boundary value problems in potential theory," Applied Numerical Mathematics, vol. 43, no. 1-2, pp. 161-173, 2002.

[75] A. Kirsch, "Characterization of the shape of a scattering obstacle using the spectral data of the far field operator," Inverse Problems, vol. 14, no. 6, pp. 1489-1512, 1998.

[76] A. Kirsch and N. Grinberg, The Factorization Method for Inverse Problems, vol. 36 of Oxford Lecture Series in Mathematics and its Applications, Oxford University Press, Oxford, UK, 2008.

[77] R. Potthast, "Stability estimates and reconstructions in inverse acoustic scattering using singular sources," Journal of Computational and Applied Mathematics, vol. 114, no. 2, pp. 247-274, 2000.

[78] M. Ikehata, "Reconstruction of obstacle from boundary measurements," Wave Motion, vol. 30, no. 3, pp. 205-223, 1999.

[79] M. Ikehata, "Reconstruction of an obstacle from the scattering amplitude at a fixed frequency," Inverse Problems, vol. 14, no. 4, pp. 949-954, 1998.

[80] M. Ikehata, "Enclosing a polygonal cavity in a two-dimensional bounded domain from Cauchy data," Inverse Problems, vol. 15, no. 5, pp. 1231-1241, 1999.

[81] M. Ikehata, "Reconstruction of a source domain from the Cauchy data," Inverse Problems, vol. 15, no. 2, pp. 637-645, 1999.

[82] D. R. Luke and R. Potthast, "The no response test-a sampling method for inverse scattering problems," SIAM Journal on Applied Mathematics, vol. 63, no. 4, pp. 1292-1312, 2003.

[83] R. Potthast, "A set-handling approach for the no-response test and related methods," Mathematics and Computers in Simulation, vol. 66, no. 4-5, pp. 281-295, 2004.

[84] R. Potthast, "On the convergence of the no response test," SIAM Journal on Mathematical Analysis, vol. 38, no. 6, pp. 1808-1824, 2007.

[85] H. W. Engl, M. Hanke, and A. Neubauer, Regularization of Inverse Problems, vol. 375 of Mathematics and its Applications, Kluwer Academic Publishers, Dordrecht, The Netherlands, 1996. 
[86] R. Potthast, "A study on orthogonality sampling," Inverse Problems, vol. 26, no. 7, Article ID 074015, 2010.

[87] K. Ito, B. Jin, and J. Zou, "A direct sampling method to an inverse medium scattering problem," Inverse Problems, vol. 28, no. 2, Article ID 025003, 11 pages, 2012.

[88] K. Ito, B. Jin, and J. Zou, "A Two-stage method for inverse medium scattering," Journal of Computational Physics, vol. 237, pp. 211-223, 2013.

[89] R. Griesmaier, "Multi-frequency orthogonality sampling for inverse obstacle scattering problems," Inverse Problems, vol. 27, no. 8, Article ID 085005, 23 pages, 2011.

[90] D. Colton and A. Kirsch, "The determination of the surface impedance of an obstacle from measurements of the far field pattern," SIAM Journal on Applied Mathematics, vol. 41, no. 1, pp. 8-15, 1981.

[91] H. Haddar and R. Kress, "On the Fréchet derivative for obstacle scattering with an impedance boundary condition," SIAM Journal on Applied Mathematics, vol. 65, no. 1, pp. 194-208, 2004.

[92] A. W. Kedzierawski, "The determination of the surface impedance of an obstacle," Proceedings of the Edinburgh Mathematical Society. Series II, vol. 36, no. 1, pp. 1-15, 1993.

[93] I. Akduman and R. Kress, "Direct and inverse scattering problems for inhomogeneous impedance cylinders of arbitrary shape," Radio Science, vol. 38, no. 3, pp. 1055-1064, 2003.

[94] R. Kress, F. Yaman, A. Yapar, and I. Akduman, "Inverse scattering for an impedance cylinder buried in a dielectric cylinder," Inverse Problems in Science and Engineering, vol. 17, no. 4, pp. 473-488, 2009.

[95] L. He, S. Kindermann, and M. Sini, "Reconstruction of shapes and impedance functions using few far-field measurements," Journal of Computational Physics, vol. 228, no. 3, pp. 717-730, 2009.

[96] R. T. Smith, "An inverse acoustic scattering problem for an obstacle with an impedance boundary condition," Journal of Mathematical Analysis and Applications, vol. 105, no. 2, pp. 333356, 1985.

[97] B. Hassen, F. B. Yosra, and H. Haddar, "Application of the linear sampling method to identify cracks with impedance boundary conditions," Inverse Problems in Science and Engineering, vol. 21, no. 2, 2013.

[98] O. Ivanyshyn and R. Kress, "Inverse scattering for surface impedance from phase-less far field data," Journal of Computational Physics, vol. 230, no. 9, pp. 3443-3452, 2011.

[99] F. Yaman, "Numerical solution of an inverse conductive boundary value problem," Radio Science, vol. 43, no. 6, Article ID RS6004, 2008.

[100] F. Yaman, "Inverse scattering of an arbitrarily shaped buried scatterer with conductive boundary condition," in Proceedings of the 8th World Congress on Computational Mechanics and 5th European Congress on Computational Methods in Applied Sciences and Engineering, Venice, Italy, 2008.

[101] F. Yaman, "A combination of shape and conductivity function reconstruction methods for an inverse boundary value problem," Wave Motion, vol. 47, no. 4, pp. 253-263, 2010.

[102] K. Aki and P. G. Richards, Quantitative Seimology: Theory and Methods, vol. 1, W.H. Freedman and Company, San Francisco, Calif, USA, 1980.

[103] G. Herglotz, "Uber die elastizitaet der erde bei beruecksichtigung ihrer variablen dichte," Zeitschrift für Mathematik und Physik, vol. 52, pp. 275-299, 1905.
[104] E. Wiechert and K. Zoeppritz, "Uber Erdbebenwellen," Nachrichten von der Königlichen Gesellschaft der Wissenschaften und von der Georg-Augusts-Universitat, vol. 4, pp. 415-549, 1907.

[105] M. L. Gerver and V. M. Markushevich, "Investigation of nonuniqueness under determining of a seismic wave propagation velocity from godo-graph," Doklady Akademii Nauk SSSR, vol. 163, no. 6, pp. 1377-1380, 1965 (Russian).

[106] M. M. Lavrent'ev and V. G. Romanov, "Three linearized inverse problems for hyperbolic equations," Doklady Akademii Nauk SSSR, vol. 171, pp. 1279-1281, 1966 (Russian).

[107] V. G. Romanov, "On reconstruction of a function from integrals along a family of curves," Siberian Mathematical Journal, vol. 10, no. 6, pp. 1364-1474, 1969.

[108] Yu. E. Anikonov, Some Methods of Investigation of Multidimensional Inverse Problems for Differential Equations, Nauka, Novosibirsk, Russia, 1978.

[109] A. L. Bukhgeim, Volterra Equations and Inverse Problems, Nauka, Novosibirsk, Russia, 1983.

[110] R. G. Mukhometov, “On one problem of Riemann's metric reconstruction," Siberian Mathematical Journal, vol. 22, no. 3, pp. 119-135, 1981.

[111] G. Uhlmann, “Travel time tomography," Journal of the Korean Mathematical Society, vol. 38, no. 4, pp. 711-722, 2001.

[112] R. G. Muhometov and V. G. Romanov, "On the problem of finding an isotropic Riemannian metric in an $n$-dimensional space," Doklady Akademii Nauk SSSR, vol. 243, no. 1, pp. 41-44, 1978 (Russian).

[113] I. N. Berntein and M. L. Gerver, "On problem of integral geometry for family of geodesic lines and an inverse kinematic problems of seismics," Doklady Akademii Nauk SSSR, vol. 243, pp. 302-306, 1978 (Russian).

[114] V. A. Sharafutdinov, Integral Geometry of Tensor Fields, Inverse and Ill-posed Problems Series, VSP, Utrecht, The Netherland, 1994.

[115] V. A. Sharafutdinov, "Finiteness theorem for the ray transform on a Riemannian manifold," Inverse Problems, vol. 11, no. 5, pp. 1039-1050, 1995.

[116] P. Stefanov and G. Uhlmann, "Rigidity for metrics with the same lengths of geodesics," Mathematical Research Letters, vol. 5, no. 1-2, pp. 83-96, 1998.

[117] V. Sharafutdinov and G. Uhlmann, "On deformation boundary rigidity and spectral rigidity of Riemannian surfaces with no focal points," Journal of Differential Geometry, vol. 56, no. 1, pp. 93-110, 2000.

[118] Yu. E. Anikonov, V. V. Bogdanov, E. Yu. Derevtsov, V. L. Miroshnichenko, N. B. Pivovarova, and L. B. Slavina, "Some approaches to a numerical solution for the multidimensional inverse kinematic problem of seismics with inner sources," Journal of Inverse and Ill-Posed Problems, vol. 17, no. 3, pp. 209238, 2009.

[119] Yu. E. Anikonov, Yu. S. Volkov, S. B. Gorshkalev, Yu. E. Derevtsov, and S. V. Mal'tseva, "On a horizontal homogeneity criterion in the inverse kinematic problem of seismic," Vestnik Novosibirskogo Gosudarstvennogo Universiteta. Seriya: Matematika, Mekhanika, Informatika, vol. 11, no. 3, pp. 3-19, 2011 (Russian).

[120] V. G. Yakhno, "A two-dimensional inverse problem for a system of dynamical Lamé equations," Soviet Physics Doklady, vol. 34, no. 7, pp. 616-617, 1989.

[121] V. G. Yakhno, "A multidimensional inverse dynamic problem of isotropic elasticity," Soviet Physics Doklady, vol. 34, no. 1, pp. 35-36, 1989. 
[122] V. G. Yakhno, Inverse Problems for Differential Equations of Elasticity, Nauka, Novosibirsk, Russia, 1990.

[123] V. G. Yakhno, "Multidimensional inverse problems for hyperbolic equations with point sources," in Ill-Posed and Inverse Problems, pp. 443-468, VSP, Zeist, The Netherlands, 2002.

[124] A. S. Alekseev, "Inverse dynamic seismic problems," in Some Methods and Algorithms for Interpretation of Geophysical Data, pp. 9-84, Nauka, Moscow, Russia, 1967.

[125] A. S. Alekseev and V. I. Dobrinsky, "Some questions of applying inverse dynamical seismology problem," Mathematical Problems in Geophysics, vol. 6, no. 2, pp. 7-53, 1975 (Russian).

[126] G. Alessandrini, "Stable determination of conductivity by boundary measurements," Applicable Analysis, vol. 27, no. 1-3, pp. 153-172, 1988.

[127] A. Alessio and P. Kinahan, "PET image reconstruction," in Nuclear Medicine, R. E. Henkin, D. Bova, G. L. Dillehay, S. M. Karesh, J. R. Halama, and R. H. Wagner, Eds., Elsevier, Philadelphia, Pa, USA, 2nd edition, 2006.

[128] S. Amari, "Dynamics of pattern formation in lateral-inhibition type neural fields," Biological Cybernetics, vol. 27, no. 2, pp. 7787, 1977.

[129] H. Ammari, E. Iakovleva, and D. Lesselier, "A MUSIC algorithm for locating small inclusions buried in a half-space from the scattering amplitude at a fixed frequency," Multiscale Modeling \& Simulation, vol. 3, no. 3, pp. 597-628, 2005.

[130] H. Ammari, G. Bao, and J. L. Fleming, "An inverse source problem for Maxwell's equations in magnetoencephalography," SIAM Journal on Applied Mathematics, vol. 62, no. 4, pp. 1369$1382,2002$.

[131] H. Ammari, An Introduction to Mathematics of Emerging Biomedical Imaging, vol. 62 of Mathématiques \& Applications, Springer, Berlin, Germany, 2008.

[132] J. L. Anderson, "An ensemble adjustment Kalman filter for data assimilation," Monthly Weather Review, vol. 129, no. 12, pp. 2884-2903, 2001.

[133] B. D. O. Anderson and J. B. Moore, Optimal Filtering, Prentice Hall, New York, NY, USA, 1979.

[134] H. J. Andrew, Stochastic Processes and Filtering Theory, Academic Press, New York, NY, USA, 1970.

[135] S. R. Arridge, "Optical tomography in medical imaging," Inverse Problems, vol. 15, no. 2, pp. R41-R93, 1999.

[136] D. C. Barber and B. H. Brown, "Applied potential tomography," Journal of Physics E, vol. 17, no. 9, article no. 002, pp. 723-733, 1984.

[137] J. S. Barlow, "Computerized clinical electroencephalography in perspective," IEEE Transactions on Biomedical Engineering, vol. 26, no. 7, pp. 377-391, 1979.

[138] R. Bayford and A. Tizzard, "Bioimpedance imaging: an overview of potential clinical applications," Analyst, vol. 137, pp. 4635-4643, 2012.

[139] P. b. Graben, D. Pinotsis, D. Saddy, and R. Potthast, "Language processing with dynamic fields," Cognitive Neurodynamics, vol. 2, no. 2, pp. 78-88, 2008.

[140] P. b. Graben and R. Potthast, "Inverse problems in dynamic cognitive modeling," Chaos, vol. 19, no. 1, Article ID 015103, 21 pages, 2009.

[141] P. b. Graben and R. Potthast, "A dynamic field account to language- related brain potentials," in Principles of Brain Dynamics: Global State Interactions, M. Rabinovich, K. Friston, and P. Varona, Eds., MIT Press, Cambridge, Mass, USA, 2012.
[142] M. I. Belishev and S. A. Ivanov, "Reconstruction of the parameters of a system of connected beams from dynamic boundary measurements," Zapiski Nauchnykh Seminarov (POMI), vol. 324, pp. 20-42, 2005.

[143] M. Bellassoued, O. Imanuvilov, and M. Yamamoto, "Inverse problem of determining the density and two Lamé coefficients by boundary data," SIAM Journal on Mathematical Analysis, vol. 40, no. 1, pp. 238-265, 2008.

[144] M. Bertero and M. Piana, "Inverse problems in biomedical imaging: modeling and methods of solution," in Complex Systems in Biomedicine, A. Quarteroni, L. Formaggia, and A. Veneziani, Eds., pp. 1-33, Springer, Berlin, Germany, 2006.

[145] G. Beylkin, "The inversion problem and applications of the generalized Radon transform," Communications on Pure and Applied Mathematics, vol. 37, no. 5, pp. 579-599, 1984.

[146] G. Beylkin and R. Burridge, "Linearized inverse scattering problems in acoustics and elasticity," Wave Motion, vol. 12, no. 1, pp. 15-52, 1990.

[147] A. S. Blagoveschenskii, "On the inverse problem in the theory of propagation of seismic waves," Problems of Mathematical Physics, vol. 1, pp. 68-81, 1966 (Russian).

[148] J. C. Bolomey and C. Pichot, "Microwave tomography: from theory to practical imaging systems," International Journal of Imaging Systems and Technology, vol. 2, pp. 144-156, 1990.

[149] J. Bolomey Ch., A. Izadnegahdar, and L. Jofre, "Microwave diffraction tomography for biomedical applications," IEEE Transactions on Microwave Theory and Techniques, vol. 30, no. 11, pp. 1998-2000, 1982.

[150] M. Bonnet and A. Constantinescu, "Inverse problems in elasticity," Inverse Problems, vol. 21, no. 2, pp. R1-R50, 2005.

[151] L. Borcea, "Electrical impedance tomography," Inverse Problems, vol. 18, no. 6, pp. R99-R136, 2002.

[152] L. Borcea and G. C. Papanicolaou, "Low frequency electromagnetic fields in high contrast media," in Surveys on Solution Methods for Inverse Problems, D. Colton, H. W. Engl, A. Louis, J. R. McLaughlin, and W. Run-dell, Eds., pp. 195-233, Springer, Vienna, Austria, 2000.

[153] L. Bourgeois, C. Chambeyron, and S. Kusiak, "Locating an obstacle in a 3D finite depth ocean using the convex scattering support," Journal of Computational and Applied Mathematics, vol. 204, no. 2, pp. 387-399, 2007.

[154] W. E. Boyse and J. B. Keller, "Inverse elastic scattering in three dimensions," Acoustical Society of America. Journal, vol. 79, no, 2, pp. 215-218, 1986.

[155] M. Brühl, M. Hanke, and M. S. Vogelius, "A direct impedance tomography algorithm for locating small inhomogeneities," Numerische Mathematik, vol. 93, no. 4, pp. 635-654, 2003.

[156] W. R. Breckon, Image reconstruction in electrical impedance tomography [Ph.D. thesis], Oxford Brookes Polytechnic, 1990.

[157] C. E. A. Brett, K. F. Lam, K. J. H. Law, D. S. McCormick, M. R. Scott, and A. M. Stuart, "Accuracy and stability of filters for dissipative PDEs," Physica D, vol. 245, pp. 34-45, 2013.

[158] C. E. A. Brett, K. F. Lam, K. J. H. Law, D. S. McCormick, M. R. Scott, and A. M. Stuart, "Stability of filters for the Navier-Stokes equation," http://arxiv.org/abs/1110.2527.

[159] K. P. Bube and R. Burridge, "The one-dimensional inverse problem of reflection seismology," SIAM Review, vol. 25, no. 4, pp. 497-559, 1983.

[160] A. L. Bukhgeim and M. V. Klibanov, "Global uniqueness of class of multidimensional inverse problems," Soviet Mathematics Doklady, vol. 24, pp. 244-247, 1981. 
[161] G. Burgers, P. J. Van Leeuwen, and G. Evensen, "Analysis scheme in the ensemble Kalman filter," Monthly Weather Review, vol. 126, no. 6, pp. 1719-1724, 1998.

[162] Y. Cai, K. Shen, and J. Wang, "Application of Radon transform in CT image matching," in Proceedings of the 16th World Conference on Non-Destructive Testing (WCNDT '04), p. 347, 2004.

[163] A. P. Calderon, "On an inverse boundary value problem," in Proceedings of the Seminar on Numerical Analysis and its applications to Continuum Physics, pp. 65-73, Sociedade Brasileira de Matemática, Rio de Janeiro, Brazil, 1980.

[164] K. Chadan and P. C. Sabatier, Inverse Problems in Quantum Scattering Theory, Springer, New York, NY, USA, 1977.

[165] N. R. Chapman, "Inverse problems in underwater acoustics," in Handbook of Signal Processing in Acoustics, D. Havelock, S. Kuwano, and M. Vorldder, Eds., pp. 1723-1735, Springer, New York, NY, USA, 2009.

[166] M. Cheney, D. Isaacson, and J. C. Newell, "Electrical impedance tomography," SIAM Review, vol. 41, no. 1, pp. 85-101, 1999.

[167] M. Cheney, "The linear sampling method and the MUSIC algorithm," Inverse Problems, vol. 17, no. 4, pp. 591-595, 2001.

[168] Z. H. Cho, J. P. Jones, and M. Singh, Foundations of Medical Imaging, Wiley, New York, NY, USA, 1993.

[169] J. R. Cox Jr., F. M. Nolle, and R. M. Arthur, "Digital analysis of the electroencephalogram, the blood pressure wave, and the electrocardiogram," Proceedings of the IEEE, vol. 60, no. 10, pp. 1137-1164, 1972.

[170] S. Coen, "On the elastic profiles of a layered medium from reflection data," Journal of the Acoustical Society of America, vol. 70, no. 1, pp. 172-175, 1981.

[171] M. D. Collins and W. A. Kuperman, "Inverse problems in ocean acoustics," Inverse Problems, vol. 10, no. 5, pp. 1023-1040, 1994.

[172] S. Coombes, P. b. Graben, and R. Potthast, "Tutorial on neural field theory," in Neural Field Theory, P. B. Graben, S. Coombes, R. Potthast, and J. J. Wright, Eds., Springer, New York, NY, USA, 2013.

[173] P. Courtier, E. Andersson, W. Heckley et al., "The ECMWF implementation of three-dimensional variational assimilation (3D-Var). I: formulation," Quarterly Journal of the Royal Meteorological Society, vol. 124, no. 550, pp. 1783-1807, 1998.

[174] “Current and historical scores," http://www.ecmwf.int.

[175] M. Defrise, "A short reader's guide to 3D tomographic reconstruction," Computerized Medical Imaging and Graphics, vol. 25, no. 2, pp. 113-116, 2001.

[176] A. J. Devaney, "Super-resolution processing of multi-static data using time reversal and MUSIC," Journal of the Acoustical Society of America. In press.

[177] A. J. Devaney, "A filtered backpropagation algorithm for diffraction tomography," Ultrasonic Imaging, vol. 4, no. 4, pp. 336-350, 1982.

[178] A. J. Devaney, "A computer simulation study of diffraction tomography," IEEE Transactions on Biomedical Engineering, vol. 30, no. 7, pp. 377-386, 1983.

[179] A. J. Devaney, "Geophysical diffraction tomography," IEEE Transactions on Geoscience and Remote Sensing, vol. 22, no. 1, pp. 3-13, 1984.

[180] S. R. Doctor, T. E. Hall, and L. D. Reid, "SAFT-the evolution of a signal processing technology for ultrasonic testing," NDT International, vol. 19, no. 3, pp. 163-167, 1986.
[181] J. W. Dower, J. W. D. Houtekamer, J. W. D. P. L, and H. L. Mitchell, "Data assimilation using an ensemble Kalman filter technique," Monthly Weather Review, vol. 126, no. 3, pp. 796811, 1998.

[182] M. Eller, V. Isakov, G. Nakamura, and D. Tataru, "Uniqueness and stability in the Cauchy problem for Maxwell and elasticity systems," in Nonlinear Partial Differential Equations and Their Applications, vol. 31 of Studies in Mathematics and Its Applications, pp. 329-349, North-Holland, Amsterdam, The Netherlands, 2002.

[183] G. Evensen, "Sequential data assimilation with a nonlinear quasi-geostrophic model using Monte Carlo methods to forecast error statistics," Journal of Geophysical Research, vol. 99, no. 5, pp. 10-162, 1994.

[184] L. D. Faddeev, "Inverse problem of quantum scattering theory. II," Journal of Soviet Mathematics, vol. 5, no. 3, pp. 334-396, 1976.

[185] E. C. Fear, S. C. Hagness, P. M. Meaney, M. Okoniewski, and M. A. Stuchly, "Enhancing breast tumor detection with near-field imaging," IEEE Microwave Magazine, vol. 3, no. 1, pp. 48-56, 2002.

[186] V. A. Feofanova and V. G. Yakhno, "Computation of the elastic moduli and density of a two-layer elastic medium using geophysical data on a surface," Soviet Mining Science, vol. 25, no. 4, pp. 327-333, 1989.

[187] V. A. Feofanova and V. G. Yakhno, "Elastic parameters of a laminar mass," Soviet Mining Science, vol. 26, no. 6, pp. 493-500, 1990.

[188] M. Freitag and R. Potthast, "Synergy of inverse problems and data assimilation techniques," in Large Scale Inverse ProblemsComputational Methods and Applications In the Earth Sciences, M. Cullen, M. A. Freitag, S. Kindermann, and R. Scheichl, Eds., vol. 13 of Radon Series on Computational and Applied Mathematics, 2013.

[189] R. P. Gilbert, T. Scotti, A. Wirgin, and Y. S. Xu, "The unidentified object problem in a shallow ocean," Journal of the Acoustical Society of America, vol. 103, no. 3, pp. 1320-1327, 1998.

[190] S. H. Gray and W. W. Symes, "Stability considerations for onedimensional inverse problems," Geophysical Journal, vol. 80, no. 1, pp. 149-163, 1985.

[191] M. Hämäläinen, R. Hari, R. J. Ilmoniemi, J. Knuutila, and O. V. Lounasmaa, "Magnetoencephalography-theory, instrumentation, and applications to noninvasive studies of the working human brain," Reviews of Modern Physics, vol. 65, no. 2, pp. 413497, 1993.

[192] E. M. Haacke, R. W. Brown, M. R. Thompson, and R. Venkatesan, Magnetic Resonance Imaging, Physical Principals and Sequence Design, John Wiley \& Son, New York, NY, USA, 1999.

[193] K.-H. Hauer, L. Kühn, and R. Potthast, "On uniqueness and non-uniqueness for current reconstruction from magnetic fields," Inverse Problems, vol. 21, no. 3, pp. 955-967, 2005.

[194] K.-H. Hauer, R. Potthast, T. Wüster, and D. Stolten, "Magnetotomography-a new method for analysing fuel cell performance and quality," Journal of Power Sources, vol. 143, no. 1-2, pp. 67-74, 2005.

[195] K.-H. Hauer and R. Potthast, "Magnetic tomography for fuel cells-current status and problems," Journal of Physics, vol. 73, no. 1, Article ID 012008, p. 17, 2007.

[196] C. J. Hellier, Handbook of Nondestructive Evaluation, McGrawHill, New York, NY, USA, 2003.

[197] D. Holder, Ed., Electrical Impedance Tomography: Methods, History and Applications, Institute of Physics Publishing, 2004. 
[198] S. Hou, K. Solna, and H. Zhao, "A direct imaging algorithm for extended targets," Inverse Problems, vol. 22, no. 4, pp. 1151-1178, 2006.

[199] G. N. Hounsfield, "Computerized transverse axial scanning (tomography): I. Description of system," British Journal of Radiology, vol. 46, no. 552, pp. 1016-1022, 1973.

[200] P. L. Houtekamer and H. L. Mitchell, "A sequential ensemble Kalman filter for atmospheric data assimilation," Monthly Weather Review, vol. 129, no. 1, pp. 123-137, 2001.

[201] B. R. Hunt, E. J. Kostelich, and I. Szunyogh, "Efficient data assimilation for spatiotemporal chaos: a local ensemble transform Kalman filter," Physica D, vol. 230, no. 1-2, pp. 112-126, 2007.

[202] M. Ikehata, "Inversion formulas for the linearized problem for an inverse boundary value problem in elastic prospection," SIAM Journal on Applied Mathematics, vol. 50, no. 6, pp. 16351644, 1990.

[203] M. Ikehata, G. Nakamura, and M. Yamamoto, "Uniqueness in inverse problems for the isotropic Lamé system," The University of Tokyo. Journal of Mathematical Sciences, vol. 5, no. 4, pp. 627692, 1998.

[204] O. Imanuvilov, V. Isakov, and M. Yamamoto, "An inverse problem for the dynamical Lamé system with two sets of boundary data," Communications on Pure and Applied Mathematics, vol. 56, no. 9, pp. 1366-1382, 2003.

[205] O. Yu. Imanuvilov and M. Yamamoto, "Carleman estimates for the non-stationary Lamé system and the application to an inverse problem," ESAIM. Control, Optimisation and Calculus of Variations, vol. 11, no. 1, pp. 1-56, 2005.

[206] O. Imanuilov and M. Yamamoto, "Carleman estimates for the non- stationary Lame system and the application to an inverse problem," in Book Control Theory of Partial Differential Equations, vol. 242 of Lectuture Notes Pure and applied Mathematics, pp. 337-374, Chapman and Hall-CRC, Boca Raton, Fla, USA, 2005.

[207] D. Isaacson and E. L. Isaacson, "Comment on Calderon's Paper: on an inverse boundary value problem," Mathematics of Computation, vol. 52, no. 186, pp. 553-559, 1989.

[208] V. Isakov, "A nonhyperbolic Cauchy problem for $\sqcup^{b} \sqcup^{c}$ and its applications to elasticity theory," Communications on Pure and Applied Mathematics, vol. 39, no. 6, pp. 747-767, 1986.

[209] J. Kaipio and E. Somersalo, Statistical and Computational Inverse Problems, vol. 160 of Applied Mathematical Sciences, Springer, New York, NY, USA, 2005.

[210] L. Jofre, M. S. Hawley, A. Broquetas, E. De Los Reyes, M. Ferrando, and A. R. Elias-Fuste, "Medical imaging with a microwave tomographic scanner," IEEE Transactions on Biomedical Engineering, vol. 37, no. 3, pp. 303-312, 1990.

[211] S. I. Kabanikhin, Inverse and Ill-Posed Problems: Theory and Applications, vol. 55 of Inverse and Ill-posed Problems Series, Walter de Gruyter, Berlin, Germany, 2012.

[212] A. C. Kak and M. Slaney, Principles of Computerized Tomographic Imaging, IEEE Press, New York, NY, USA, 1988.

[213] R. E. Kalman, "A new approach to linear filtering and prediction problems," Journal of Basic Enqineerinq, vol. 82, no. 1, pp. 35-45, 1960.

[214] A. L. Karchevsky, "Reconstruction of pressure velocities and boundaries of thin layers in thinly-stratified layers," Journal of Inverse and Ill-Posed Problems, vol. 18, no. 4, pp. 371-388, 2010.

[215] V. G. Romanov, Inverse Problems of Mathematical Physics, VNU Science Press, Utrecht, The Netherlands, 1987.
[216] E. A. Volkova and V. G. Romanov, "Inverse dynamic problems for an anisotropic elastic medium," Doklady Akademii Nauk SSSR, vol. 267, no. 4, pp. 780-783, 1982 (Russian).

[217] A. E. Yagle and B. C. Levy, "A layer stripping solution of the inverse problem for a one dimensional elastic medium," Geophysics, vol. 50, no. 3, pp. 425-433, 1985.

[218] V. G. Yakhno, "One-dimensional inverse problem of anisotropic elasticity for string sources," Soviet Physics Doklady, vol. 30, no. 11, pp. 931-932, 1985.

[219] M. Meadows and S. Coen, "Exact inversion of plane-layered isotropic and anisotrophic elastic media by the state-space approach," Geophysics, vol. 51, no. 11, pp. 2031-2050, 1986.

[220] P. E. Sacks and W. W. Symes, "The inverse problem for a fluid over a layered elastic half-space," Inverse Problems, vol. 6, no. 6, pp. 1031-1054, 1990.

[221] P. E. Sacks and V. G. Yakhno, "The inverse problem for a layered anisotropic half space," Journal of Mathematical Analysis and Applications, vol. 228, no. 2, pp. 377-398, 1998.

[222] A. G. Megrabov, Forward andInverse Problems for Hyperbolic, Elliptic, and Mixed Type Equations, Inverse and Ill-Posed Problems Series, VSP, Utrecht, The Netherlands, 2003.

[223] A. G. Ramm, Inverse Problems, Mathematical and Analytical Techniques with Applications to Engineering, Springer, New York, NY, USA, 2005.

[224] A. Morassi, G. Nakamura, and M. Sini, "An inverse dynamical problem for connected beams," European Journal of Applied Mathematics, vol. 16, no. 1, pp. 83-109, 2005.

[225] S. Nagayasu, "An inverse problem for the elastic equation in plane-stratified media," SUT Journal of Mathematics, vol. 41, no. 2, pp. 117-135, 2005.

[226] A. Morassi, G. Nakamura, K. Shirota, and M. Sini, "A variational approach for an inverse dynamical problem for composite beams," European Journal of Applied Mathematics, vol. 18, no. 1, pp. 21-55, 2007.

[227] V. G. Romanov, "Determination of the parameters of a layered piecewise-constant medium for an unknown form of an impulse source," Siberian Mathematical Journal, vol. 48, no. 6, pp. 1074-1084, 2007.

[228] V. G. Romanov, "On the problem of determining the parameters of a layered elastic medium and an impulse source," Siberian Mathematical Journal, vol. 49, no. 5, pp. 919-943, 2008.

[229] V. G. Yakhno, "The linearized inverse multidimensional problem of Lamb," Soviet Mathematics. Doklady, vol. 29, no. 3, pp. 517-520, 1984.

[230] R. H. Stolt and A. B. Weglein, "Migration and inversion of seismic data," Geophysics, vol. 50, no. 12, pp. 2458-2472, 1985.

[231] V. A. Sharafutdinov, "Quasi-isotropic approximation in dynamical elasticity and some problems of geotomography," Russian Geology and Geophysics, vol. 35, pp. 68-82, 1994.

[232] V. A. Sharafutdinov, "Linearized inverse problems of determining the parameters of transversally isotropic elastic media from measurements of refracted waves," Journal of Inverse and IllPosed Problems, vol. 4, no. 3, pp. 245-266, 1996.

[233] L. G. Steinberg, “The linearization of inverse spectral problems for nonhomogeneous elastic bodies," Inverse Problems, vol. 12, no. 4, pp. 483-497, 1996.

[234] G. Uhlmann, "Inverse boundary value problems and applications," Astérisque, vol. 207, no. 6, pp. 153-211, 1992.

[235] J. Sylvester and G. Uhlmann, "A global uniqueness theorem for an inverse boundary value problem," Annals of Mathematics, vol. 125, no. 1, pp. 153-169, 1987. 
[236] J. Sylvester and G. Uhlmann, "Inverse problems in anisotropic media," in Inverse Scattering and Applications, vol. 122 of Contemporary Mathematics, pp. 105-117, American Mathematical Society, Providence, RI, USA, 1991.

[237] G. Nakamura and G. Uhlmann, "Identification of Lamé parameters by boundary measurements," American Journal of Mathematics, vol. 115, no. 5, pp. 1161-1187, 1993.

[238] G. Nakamura and G. Uhlmann, "Global uniqueness for an inverse boundary problem arising in elasticity," Inventiones Mathematicae, vol. 118, no. 3, pp. 457-474, 1994.

[239] G. Nakamura, K. Tanuma, and G. Uhlmann, "Layer stripping for a transversely isotropic elastic medium," SIAM Journal on Applied Mathematics, vol. 59, no. 5, pp. 1879-1891, 1999.

[240] L. V. Rachele, "An inverse problem in elastodynamics: uniqueness of the wave speeds in the interior," Journal of Differential Equations, vol. 162, no. 2, pp. 300-325, 2000.

[241] C.-L. Lin and G. Nakamura, "Conditional stability for the hexagonal anisotropic elastic dynamical systems," Communications in Partial Differential Equations, vol. 34, no. 10-12, pp. 12511264, 2009.

[242] V. I. Kukulin and R. S. Mackintosh, “The application of inversion to nuclear scattering," Journal of Physics G, vol. 30, no. 2, pp. R1-R55, 2004.

[243] L. R. Lines and S. Treitel, "Tutorial: a review of least-squares inversion and its application to geophysical problems," Geophysical Prospecting, vol. 32, no. 2, pp. 159-186, 1984.

[244] R. S. Mackintosh, "Inverse scattering: applications to nuclear physics," http://arxiv.org/abs/1205.0468.

[245] R. Snieder and J. Trampert, "Inverse problems in geophysics," in Wave-Field Inversion, A. Wirgin, Ed., pp. 119-190, Springer, New York, NY, USA, 1999.

[246] S. Twomey, Introduction to the Mathematics of Inversion in Remote Sensing and Indirect Measurement, Dover, New York, NY, USA, 1996.

[247] Y. Wang, "Regularization for inverse models in remote sensing," Progress in Physical Geography, vol. 36, no. 1, pp. 38-59, 2012.

[248] C. Rozier, D. Lesselier, T. S. Angell, and R. E. Kleinman, "Shape retrieval of an obstacle immersed in shallow water from singlefrequency farfields using a complete family method," Inverse Problems, vol. 13, no. 2, pp. 487-508, 1997.

[249] T. Scotti and A. Wirgin, "Shape reconstruction of an impenetrable scattering body via the Rayleigh hypothesis," Inverse Problems, vol. 12, no. 6, pp. 1027-1055, 1996.

[250] M. Taroudakis and G. Makrakis, Eds., Inverse Problems in Underwater Acoustics, Springer, New York, NY, USA, 2001.

[251] M. I. Taroudakis, "Inverse problems in underwater acoustics," in Proceedings of the 2nd EAA International Symposium on Hydroacoustics, A. Stepnowski, R. Salamon, and E. Kozaczka, Eds., pp. 281-290, 1999.

[252] Y. Xu and Y. Yan, "Boundary integral equation method for source localization with a continuous wave sonar," Journal of the Acoustical Society of America, vol. 92, no. 2 I, pp. 995-1002, 1992.

[253] R. Prakash, Non-Destructive Testing Techniques, New Age Science, 2009.

[254] A. Seydel, "Ultrasonic synthetic aperture focusing techniques in NDT," in Research Techniques in Nondestructive Testing, R. S. Sharpe, Ed., vol. 6, The New York Academy of Sciences, New York, NY, USA, 1983.

[255] C. Pintavirooj and M. Sangworasil, "Ultrasonic diffraction tomography," The International Journal of Applied Biomedical Engineering, vol. 1, no. 1, pp. 34-40, 2008.
[256] M. Slaney, A. C. Kak, and L. E. Larsen, "Limitations of imaging with first-order diffraction tomography," IEEE Transactions on Microwave Theory and Techniques, vol. 32, no. 8, pp. 860-874, 1984.

[257] R. S. Wu and M. N. Toksoz, "Diffraction tomography and multisource holography applied to seismic imaging," Geophysics, vol. 52, no. 1, pp. 11-25, 1987.

[258] A. Kirsch, "The MUSIC algorithm and the factorization method in inverse scattering theory for inhomogeneous media," Inverse Problems, vol. 18, no. 4, pp. 1025-1040, 2002.

[259] R. Marklein, J. Miao, M. Rahman, and K. J. Langenberg, "Inverse scattering and imaging in NDT: recent applications and advances," in Proceedings of the European Conference on Non-Destructive Testing (ECNDT '06), Berlin, Germany, 2006.

[260] J. Radon, "Über die bestimmung von funktionen durch ihre integralwerte längs gewisser mannigfaltigkeiten," Berichte Uber die Verhandlun- Gen der Koniglich-Sachsischen Akademie der Wissenschaften Zu Leipzig, Mathematisch-Physische Klasse, vol. 69, pp. 262-277, 1917.

[261] S. Webb, From the Watching of Shadows: The Origins of Radiological Tomography, Adam Hilger, Bristol, New York, USA, 1990.

[262] F. Natterer, The Mathematics of Computerized Tomography, vol. 32 of Classics in Applied Mathematics, SIAM, Philadelphia, $\mathrm{Pa}$, USA, 2001.

[263] M. J. Paulus, S. S. Gleason, S. J. Kennel, P. R. Hunsicker, and D. K. Johnson, "High resolution X-ray computed tomography: an emerging tool for small animal cancer research," Neoplasia, vol. 2, no. 1-2, pp. 62-70, 2000.

[264] S. Webb, The Physics of Medical Imaging, Taylor \& Francis, New York, NY, USA, 1988.

[265] W. R. B. Lionheart, "EIT reconstruction algorithms: pitfalls, challenges and recent developments," Physiological Measurement, vol. 25, no. 1, pp. 125-142, 2004.

[266] K. Paulson, W. Breckon, and M. Pidcock, "Electrode modelling in electrical impedance tomography," SIAM Journal on Applied Mathematics, vol. 52, no. 4, pp. 1012-1022, 1992.

[267] G. J. Saulnier, R. S. Blue, J. C. Newell, D. Isaacson, and P. M. Edic, "Electrical impedance tomography," IEEE Signal Processing Magazine, vol. 18, no. 6, pp. 31-43, 2001.

[268] A. D. Seagar, D. C. Barber, and B. H. Brown, "Electrical impedance imaging," IEE Proceedings A, vol. 134, no. 2, pp. 201210, 1987.

[269] E. Niedermeyer and F. L. da Silva, Eds., Electroencephalography: Basic Principles, Clinical Applications and Related Fields, Lippincott Williams \& Wilkins, Philadelphia, Pa, USA, 5th edition, 2005.

[270] M. Teplan, "Fundamentals of EEG measurements," Measurement Science Review, vol. 2, pp. 1-11, 2002.

[271] G. Katti, S. A. Ara, and A. Shireen, "Magnetic resonance imaging (MRI) - a review," International Journal of Dental Clinics, vol. 3, no. 1, pp. 65-70, 2011.

[272] T. Schaeffter and H. Dahnke, "Magnetic resonance imaging and spectroscopy," in Molecular Imaging I, vol. 185 of Handbook of Experimental Pharmacology, pp. 75-90, Springer, Berlin, Germany, 2008.

[273] M. T. Vlaardingerbroek and J. A. Boer, Magnetic Resonance Imaging: Theory and Practice, Springer, New York, NY, USA, 3rd edition, 2003.

[274] C. Westbrook, C. K. Roth, and J. Talbot, MRI in Practice, WileyBlackwell, Oxford, UK, 4th edition, 2011. 
[275] R. M. Lewitt and S. Matej, "Overview of methods for image reconstruction from projections in emission computed tomography," Proceedings of the IEEE, vol. 91, no. 10, pp. 1588-1611, 2003.

[276] M. T. Madsen, "Recent advances in SPECT imaging," Journal of Nuclear Medicine, vol. 48, no. 4, pp. 661-673, 2007.

[277] T. F. Massoud and S. S. Gambhir, "Molecular imaging in living subjects: seeing fundamental biological processes in a new light," Genes and Development, vol. 17, no. 5, pp. 545-580, 2003.

[278] A. Rahmim and H. Zaidi, "PET versus SPECT: strengths, limitations and challenges," Nuclear Medicine Communications, vol. 29, no. 3, pp. 193-207, 2008.

[279] E. Tanaka, "Recent progress on single photon and positron emission tomography-from detectors to algorithms," IEEE Transactions on Nuclear Science, vol. NS-34, no. 1, pp. 313-320, 1986.

[280] D. W. Townsend, R. A. Isoardi, and B. Bendriem, The Theory and Practice of 3D PET, Kluwer Academic Publishers, New York, NY, USA, 1998.

[281] S. Vandenberghe, Y. D’Asseler, R. Van De Walle et al., "Iterative reconstruction algorithms in nuclear medicine," Computerized Medical Imaging and Graphics, vol. 25, no. 2, pp. 105-111, 2001.

[282] G. L. Zeng, "Image reconstruction-a tutorial," Computerized Medical Imaging and Graphics, vol. 25, no. 2, pp. 97-103, 2001.

[283] R. A. Lemons and C. F. Quate, "Acoustic microscopy: biomedical applications," Science, vol. 188, no. 4191, pp. 905-911, 1975.

[284] B. J. Roth, P. J. Basser, and J. P. Wikswo Jr., "A theoretical model for magneto-acoustic imaging of bioelectric currents," IEEE Transactions on Biomedical Engineering, vol. 41, no. 8, pp. 723$728,1994$.

[285] S. Semenov, "Microwave tomography: review of the progress towards clinical applications," Philosophical Transactions of the Royal Society of London. Series A, vol. 367, no. 1900, pp. 30213042, 2009.

[286] S. Y. Semenov, R. H. Svenson, A. E. Boulyshev et al., "Microwave tomography: two-dimensional system for biological imaging," IEEE Transactions on Biomedical Engineering, vol. 43, no. 9, pp. 869-877, 1996.

[287] S. Y. Semenov, R. H. Svenson, A. E. Bulyshev et al., "Three-dimensional microwave tomography: initial experimental imaging of animals," IEEE Transactions on Biomedical Engineering, vol. 49, no. 1, pp. 55-63, 2002.

[288] W. Tabbara, B. Duchêne, Ch. Pichot, D. Lesselier, L. Chommeloux, and N. Joachimowicz, "Diffraction tomography: contribution to the analysis of some applications in microwaves and ultrasonics," Inverse Problems, vol. 4, no. 2, pp. 305-331, 1988.

[289] L. Wang and H.-I. Wu, Biomedical Optics: Principles and Imaging, John Wiley \& Sons, New York, NY, USA, 2007.

[290] H. R. Wilson and J. D. Cowan, "Excitatory and inhibitory interactions in localized populations of model neurons," Biophysical Journal, vol. 12, no. 1, pp. 1-24, 1972.

[291] H. R. Wilson and J. D. Cowan, "A mathematical theory of the functional dynamics of cortical and thalamic nervous tissue," Kybernetik, vol. 13, no. 2, pp. 55-80, 1973.

[292] F. Yaman and S. Şimşek, "Neural Network approach to determine non smooth one-dimensional profiles in inverse scattering theory," Microwave and Optical Technology Letters, vol. 49, no. 12, pp. 3158-3162, 2007.

[293] F. Yaman and S. Şimșek, "Shape reconstruction of an inhomogeneous impedance cylinder via potential and Neural Network approach," Microwave and Optical Technology Letters, vol. 51, no. 1, pp. 119-124, 2009.
[294] R. Potthast and P. b. Graben, "Inverse problems in neural field theory," SIAM Journal on Applied Dynamical Systems, vol. 8, no. 4, pp. 1405-1433, 2009.

[295] R. Potthast and P. b. Graben, "Dimensional reduction for the inverse problem of neural held theory," Frontiers in Neuroscience, vol. 3, article 17, 2009.

[296] R. Kress, L. Kühn, and R. Potthast, "Reconstruction of a current distribution from its magnetic field," Inverse Problems, vol. 18, no. 4, pp. 1127-1146, 2002.

[297] B. A. Marx, Dynamic Magnetic Tomography, Der Andere, 2011.

[298] B. A. Marx and R. Potthast, "Data assimilation algorithms for dynamic magnetic tomography," In press.

[299] W. Lahoz, R. Swinbank, and B. Khattatov, Data Assimilation: Making Sense of Observations, Springer, New York, NY, USA, 2010.

[300] J. M. Lewis, S. Lakshmivarahan, and S. Dhall, Dynamic Data Assimilation: A Least Squares Approach, vol. 104 of Encyclopedia of Mathematics and its Applications, Cambridge University Press, Cambridge, UK, 2006.

[301] A. C. Lorenc, S. P. Ballard, R. S. Bell et al., "The Met. Office global three-dimensional variational data assimilation scheme," Quarterly Journal of the Royal Meteorological Society, vol. 126, no. 570, pp. 2991-3012, 2000.

[302] E. N. Lorenz, "Deterministic nonperiodic flow," Journal of the Atmospheric Sciences, vol. 20, no. 2, pp. 130-141, 1963.

[303] C. D. Rodgers, Inverse Methods for Atmospheric Sounding: Theory and Practice, vol. 2 of Series on Atmospheric, Oceanic and Planetary Physics, World Scientific, River Edge, NJ, USA, 2000.

[304] A. M. Stuart, “Inverse problems: a Bayesian perspective," Acta Numerica, vol. 19, pp. 451-559, 2010.

[305] J. S. Whitaker and T. M. Hamill, "Ensemble data assimilation without perturbed observations," Monthly Weather Review, vol. 130, no. 7, pp. 1913-1924, 2002.

[306] E. Ott, B. R. Hunt, I. Szunyogh et al., "A local ensemble Kalman filter for atmospheric data assimilation," Tellus A, vol. 56, no. 5, pp. 415-428, 2004.

[307] I. Szunyogh, E. J. Kostelich, G. Gyarmati et al., "Assessing a local ensemble Kalman filter: perfect model experiments with the national centers for environmental prediction global model," Tellus A, vol. 57, no. 4, pp. 528-545, 2005.

[308] I. Szunyogh, E. J. Kostelich, G. Gyarmati et al., "A local ensemble transform Kalman filter data assimilation system for the NCEP global model,” Tellus A, vol. 60, no. 1, pp. 113-130, 2008.

[309] B. A. Marx and R. Potthast, "On instabilities in data assimilation algorithms," GEM-International Journal on Geomathematics, vol. 3, no. 2, pp. 253-278, 2012.

[310] R. W. E. Potthast, A. J. F. Moodey, A. S. Lawless, and P. J. van Leeuwen, "On error dynamics and instability in data assimilation," MPS-2012-05, In press, http://www.reading.ac.uk/mathsand-stats/research/maths-preprints.aspx.

[311] A. J. F. Moodey, A. S. Lawless, R. W. E. Potthast, and P. J. van Leeuwen, "Nonlinear error dynamics for cycled data assimilation methods," Inverse Problems, vol. 29, no. 2, Article ID 025002, 18 pages, 2013. 


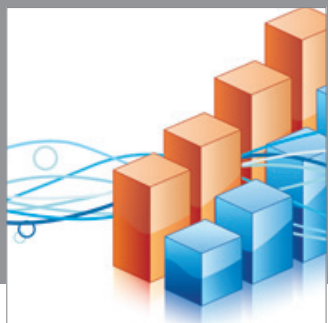

Advances in

Operations Research

mansans

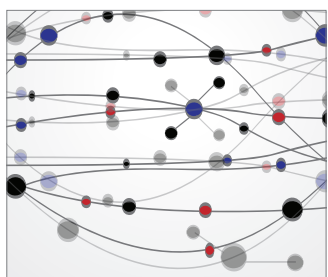

The Scientific World Journal
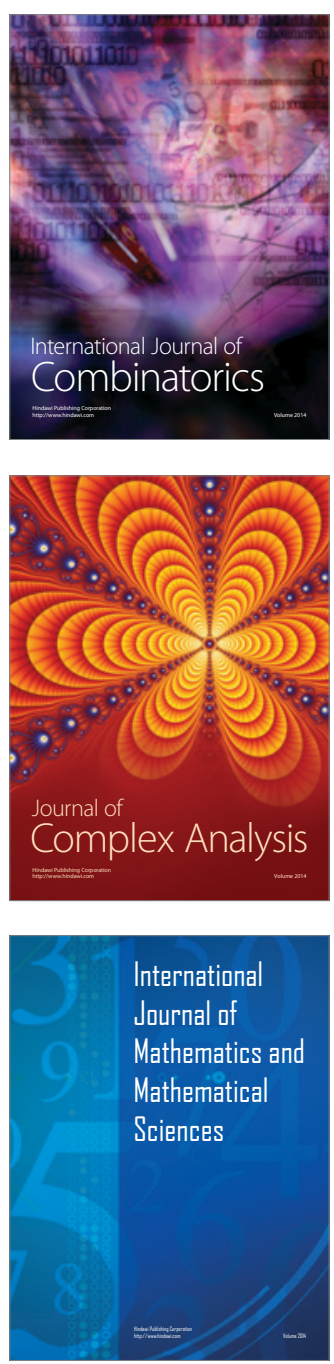
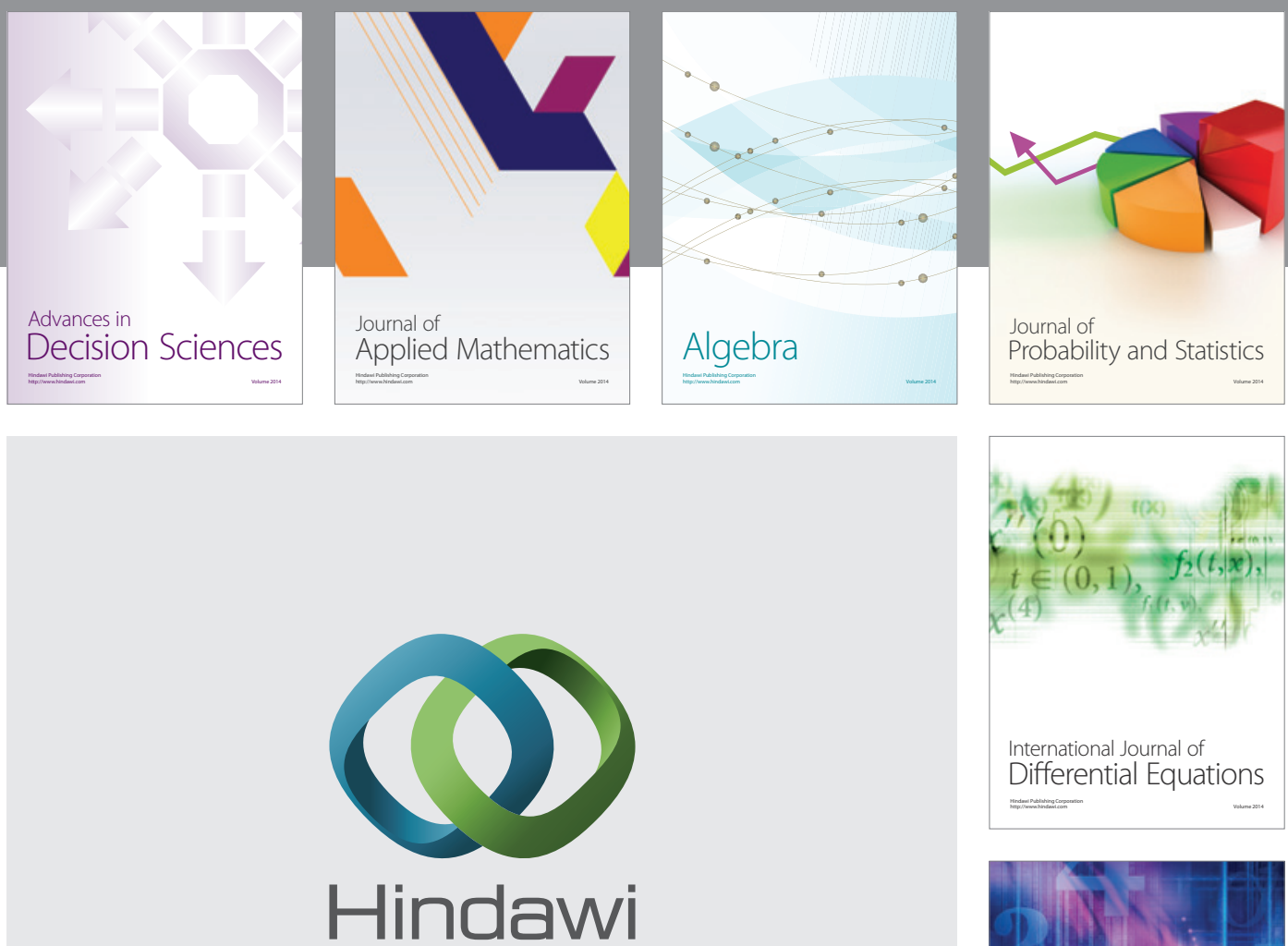

Submit your manuscripts at http://www.hindawi.com
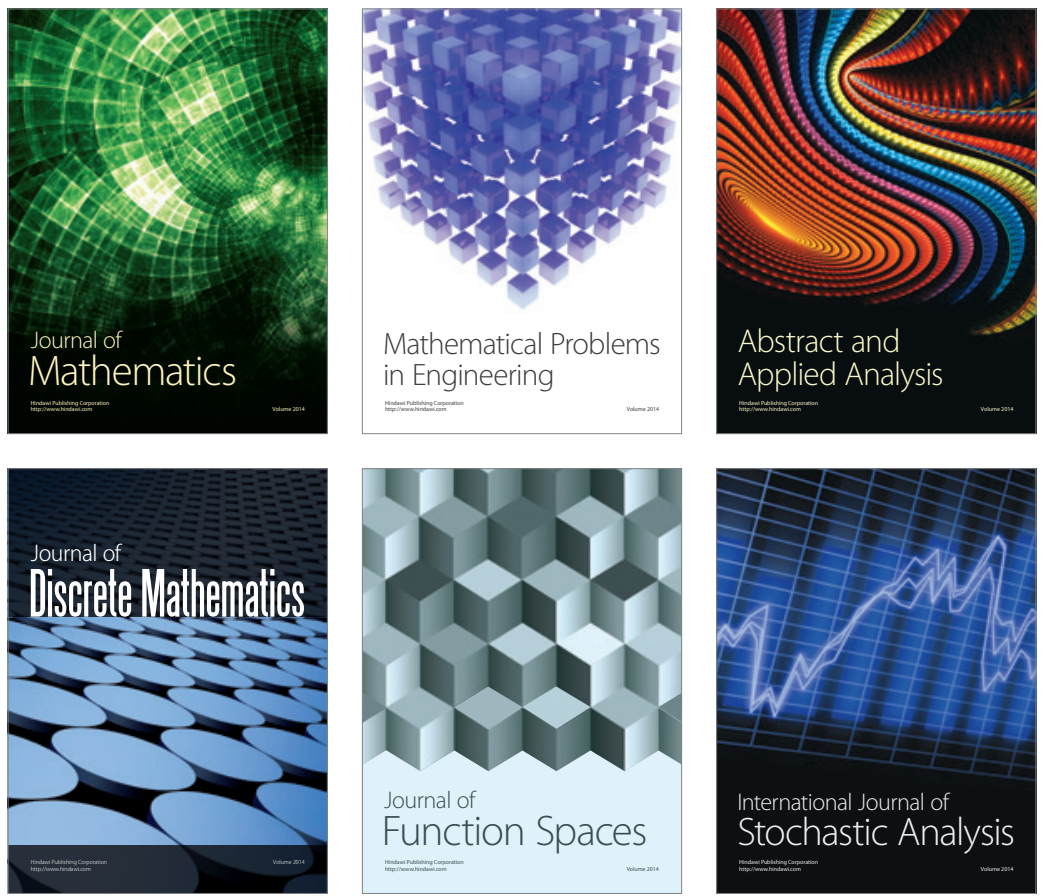

Journal of

Function Spaces

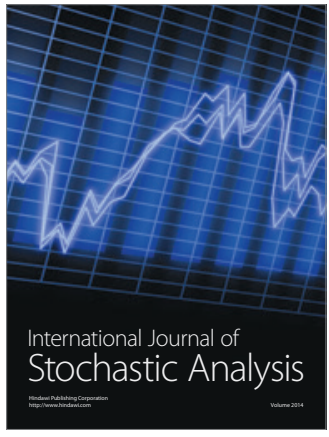

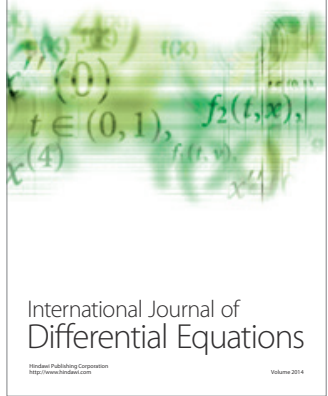
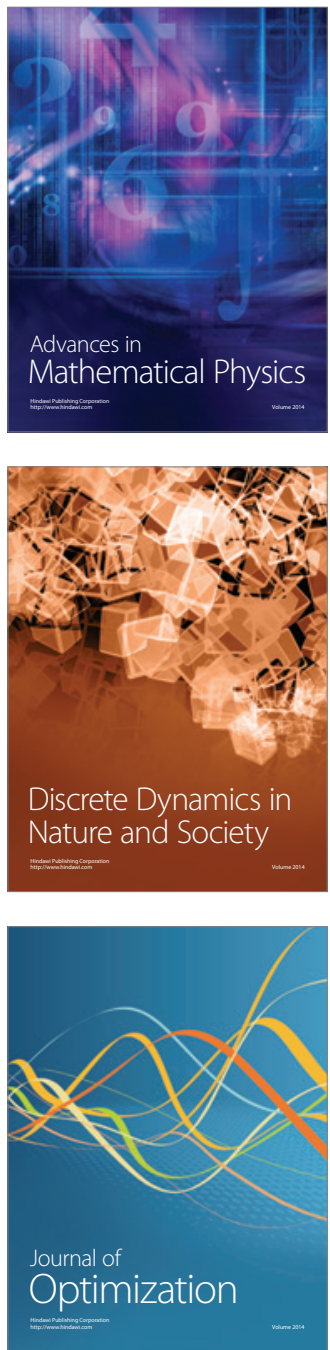